\title{
Farnesal-loaded pH-sensitive polymeric micelles provided effective prevention and treatment on dental caries
}

\author{
Youping $\mathrm{Yi}^{\dagger}$, Lujun Wang ${ }^{\dagger}$, Lin Chen ${ }^{\dagger}$, Yan Lin ${ }^{\dagger}$, Zhongling Luo, Zhenyu Chen, Ting Li, Jianming Wu ${ }^{*}$ \\ and Zhirong Zhong* ${ }^{*}$
}

\begin{abstract}
Background: Farnesol is a sesquiterpene from propolis and citrus fruit that shows promising anti-bacterial activity for caries treatment and prevention, but its hydrophobicity limits the clinical application. We aimed to develop the novel polymeric micelles (PMs) containing a kind of derivative of farnesol and a ligand of pyrophosphate (PPi) that mediated PMs to adhere tightly with the tooth enamel.

Results: Farnesal (Far) was derived from farnesol and successfully linked to PEG via an acid-labile hydrazone bond to form PEG-hyd-Far, which was then conjugated to PPi and loaded into PMs to form the aimed novel drug delivery system, PPi-Far-PMs. The in vitro test about the binding of PPi-Far-PMs to hydroxyapatite showed that PPi-Far-PMs could bind rapidly to hydroxyapatite and quickly release Far under the acidic conditions. Results from the mechanical testing and the micro-computed tomography indicated that PPi-Far-PMs could restore the microarchitecture of teeth with caries. Moreover, PPi-Far-PMs diminished the incidence and severity of smooth and sulcal surface caries in rats that were infected with Streptococcus mutans while being fed with a high-sucrose diet. The anti-caries efficacy of free Far can be improved significantly by PPi-Far-PMs through the effective binding of it with tooth enamel via PPi.
\end{abstract}

Conclusions: This novel drug-delivery system may be useful for the treatment and prevention of dental caries as well as the targeting therapy of anti-bacterial drugs in the oral disease.

Keywords: pH-sensitive, Polymeric micelles, Hydroxyapatite, Farnesal, Dental caries

\section{Introduction}

Dental caries is one of the most prevalent preventable diseases, and its incidence is especially high among young people. Caries progress when bacteria metabolize fermentable carbohydrates to produce acid, which dissolves the hydroxyapatite in teeth [1]. One promoter of caries progression is dental plaque, the complex multispecies biofilm formed by Streptococcus mutans and

\footnotetext{
*Correspondence: jianmingwu@swmu.edu.cn; zhongzr@swmu.edu.cn

${ }^{\dagger}$ Youping Yi, Lujun Wang, Lin Chen and Yan Lin contributed equally to this work

Department of Pharmaceutical Sciences, School of Pharmacy, Southwest Medical University, Luzhou, Sichuan 646000, China
}

other organisms that colonize the surface of teeth [2, 3]. S. mutans can efficiently convert dietary sucrose into extracellular polysaccharides that promote $S$. mutans accumulation and form a dense matrix that protects the embedded bacteria $[4,5]$. S. mutans ferments sucrose within the polysaccharide matrix, and creates a highly acidic microenvironment [6-8], with $\mathrm{pH}$ falling as low as $\mathrm{pH} 4.5$ [9]. This acidity dissolves the enamel, initiating caries, which can lead to tooth loss, pain and infections [10-12].

Broad-spectrum antimicrobials such as chlorhexidine and triclosan can kill or inhibit the growth of cariogenic bacteria, but their poor selectivity means that they may 
disturb the normal balance of microflora in the oral cavity. Chlorhexidine may also stain teeth and cause calculus formation [10]. Fluoride products can prevent dental caries through promoting the remineralization process, but they carry the risk of dental fluorosis, for example, overuse can affect children's bone development. Sugar substitutes can exert anti-caries effects but large amounts of them may be required to achieve clinical efficacy [10, 13]. Several natural products and their derivatives show potential against $S$. mutans, such as green tea [14], citrus lemon oil [15], and Galla chinensis [16].

One of the natural products with the proven anti-caries efficacy is farnesol (3,7,11-trimethyl-2,6,10-dodecatrien-1-ol) in propolis and citrus fruit essential oil, et al. Farnesol increases the permeability of bacterial membranes to protons and reduces glycolytic activity of S. mutans in biofilms $[17,18]$, it inhibits the growth of Staphylococcus aureus [19] and Staphylococcus epidermidis [20], and also inhibits Candida albicans biofilm formation [21]. Our preliminary studies have shown that farnesal (Far; 3,7,11-trimethyl-2,6,10-dodecatrienal), the derivative of farnesol, could inhibit $S$. mutans growth, but it is quite hydrophobic. In the present work, we wished to design a delivery system for Far that would compensate for these disadvantages.

We solubilized Far by linking it to hydrophilic PEG and made this linkage acid-sensitive by conjugating Far to the hydrazine groups of PEG via hydrazone bonds. In this way, we aimed to make Far more selective because it would be released selectively in acidic, cariogenic microenvironments in the oral cavity. We added Tris(tetra$n$-butylammonium) hydrogen pyrophosphate (TBAP) to the PEG-hyd-Far amphiphilic conjugate, since the pyrophosphate (PPi) moiety binds rapidly to hydroxyapatite $\left[\mathrm{Ca}_{10}\left(\mathrm{PO}_{4}\right)_{6}(\mathrm{OH})_{2}\right][22,23]$, in which hydroxyapatite accounts for $95-96 \%$ of the enamel mass [24, 25]. Finally, we inserted the entire conjugate into polymeric micelles (PMs) to further solubilize Far and enhance its bioavailability. Results from in vitro and in vivo rat model of the induced caries suggested that this drug-delivery platform could substantially improve the anti-caries efficacy of Far.

\section{Results}

\section{Synthesis of pH-sensitive dentotropic PPi-PEG-hyd-Far polymeric conjugate}

To develop a pH-sensitive dentotropic polymeric conjugate for the formulations of polymeric micelles, we synthesized the PPi-PEG-hyd-Far according to the synthetic scheme in Fig. 1. The following results from ${ }^{1} \mathrm{H}$ and ${ }^{13} \mathrm{C}$ NMR confirmed the synthesis of PPi-PEG-hyd-Far as described below.

Compound 1 (Additional file 1: Figure S1) gave a ${ }^{1} \mathrm{H}$ NMR spectrum (400 MHz, Chloroform-d) showing $\delta$ (ppm) 7.01 (s, 1H), 6.67 (s, 1H), 4.27 (s, 2H), 3.64 (s, $180 \mathrm{H}), 1.45$ (s, 9H). The ${ }^{13} \mathrm{C}$ NMR (101 MHz, Chloroform-d) showed $\delta$ (ppm) 70.71, 28.40. Compound 2 (Additional file 1: Figure S2) gave a ${ }^{1} \mathrm{H}$ NMR spectrum (400 MHz, Chloroform-d) showing $\delta$ (ppm) 4.30-4.19 (m, 4H), 3.87 (s, 1H), $3.62(\mathrm{~s}, 180 \mathrm{H}), 2.45$ (s, 2H), 1.44 (s, 9H). The ${ }^{13} \mathrm{C}$ NMR spectrum $(101 \mathrm{MHz}$, Chloroform-d) showed $\delta$ (ppm) 155.82, 70.67, 69.36, 68.88, 65.47, 64.93, 28.37. The ${ }^{31} \mathrm{P}$ NMR spectrum $\left(\mathrm{D}_{2} \mathrm{O}\right.$, $160 \mathrm{MHz}$ ) showed $\delta$ (ppm) -9.49 (m, 2P). Compound 3 (Additional file 1: Figure S3) gave a ${ }^{1} \mathrm{H}$ NMR spectrum (400 MHz, Chloroform-d) showing $\delta$ (ppm) 6.27 (s, $1 \mathrm{H}), 4.27-4.21(\mathrm{~m}, 4 \mathrm{H}), 3.65$ (s, 180H), 1.87 (s, 3H). The ${ }^{13} \mathrm{C}$ NMR spectrum (101 MHz, Chloroform-d) showed $\delta$ (ppm) 70.72, 69.60, 64.71. Compound 4 (Additional file 1: Figure S4) gave an ${ }^{1} \mathrm{H}$ NMR spectrum $(400 \mathrm{MHz}$, Chloroform-d) showing $\delta(\mathrm{ppm}) 8.63(\mathrm{~s}, 1 \mathrm{H}), 7.80(\mathrm{~m}$, $1 \mathrm{H}), 6.08(\mathrm{~s}, 1 \mathrm{H}), 5.07(\mathrm{~m}, 2 \mathrm{H}), 4.33$ (s, 3H), $3.63(\mathrm{~s}$, $182 \mathrm{H}), 2.21(\mathrm{~s}, 2 \mathrm{H}), 2.12(\mathrm{~s}, 3 \mathrm{H}), 2.03(\mathrm{~s}, 2 \mathrm{H}), 1.96(\mathrm{~s}$, $2 \mathrm{H}), 1.85-1.82(\mathrm{~m}, 2 \mathrm{H}), 1.66(\mathrm{~s}, 3 \mathrm{H}), 1.57$ (s, 6H). The ${ }^{13} \mathrm{C}$ NMR spectrum (101 MHz, Chloroform-d) showed $\delta(\mathrm{ppm}) 136.05,131.67,124.40,124.29,123.32,123.13$, 72.80, 70.70, 70.40, 69.48, 40.20, 39.84, 26.84, 26.21, 25.90, 17.89, 17.47, 16.20.

\section{Characteristics of polymeric micelles}

The pH-sensitive dentotropic PPi-PEG-hyd-Far and $\mathrm{mPEG}_{2000}-\mathrm{PLA}_{2000}$ polymeric conjugate were self-assembled into PPi-Far-PMs using a film hydration procedure. Dynamic light scattering showed the resulting blank PMs to have an average diameter of $15.95 \pm 0.10 \mathrm{~nm}$ with a polydispersity index of $0.086 \pm 0.008$ (Fig. 2a) and zeta potential of $-0.71 \pm 0.07 \mathrm{mV}$. PPi-Far-PMs were much larger, with a diameter of $146.20 \pm 0.87 \mathrm{~nm}$, polydispersity index of $0.234 \pm 0.012$ (Fig. 2b) and zeta potential of $-4.92 \pm 0.24 \mathrm{mV}$. Far-PMs and PPi-Far-PMs were similar in size and zeta potential. In all preparations, the polydispersity index was less than 0.3 , suggesting particle uniformity. Consistent with this idea, transmission electron microscopy showed PMs and PPi-Far-PMs to be uniformly spherical (Fig. 2c, d). The CMCs of blank PMs and PPi-Far-PMs were, respectively, $2.76 \times 10^{-3}$ and $7.92 \times 10^{-4} \mathrm{mg} / \mathrm{mL}$ (Additional file 1: Figure S5A, B).

We developed an HPLC method to calculate drug loading and encapsulation efficiencies of the PMs. The retention time of Far in PMs was consistent with that of the Far standard, and no interference signals were observed near the characteristic peak. The Far standard curve was linear over the range of $10-400 \mu \mathrm{g} / \mathrm{mL}$ (Abs $=15,146 *$ Conc$\left.29,860, R^{2}=0.9992\right)$. Intra- and inter-day relative standard deviation (RSD) was less than $10 \%$, and Far extraction recoveries were $99.09 \pm 0.48 \%$ (RSD, $0.48 \%$ ) at low concentrations, $99.84 \pm 0.10 \%$ (RSD, $0.10 \%$ ) at medium 


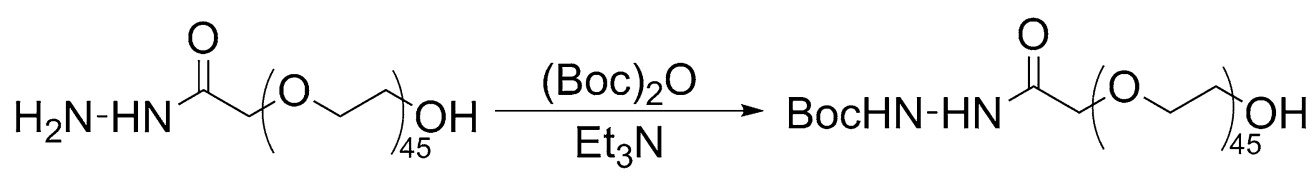

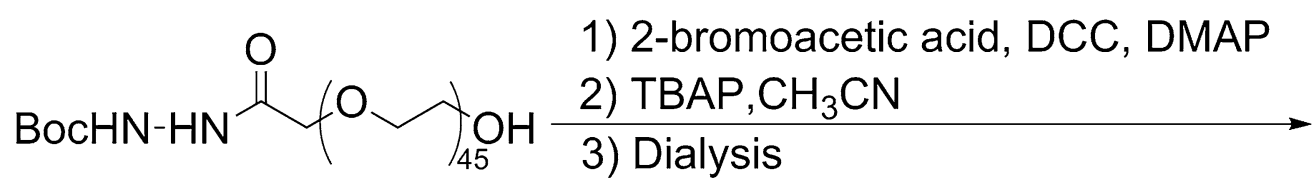<smiles>CC(C)OCCOC(=O)COP(=O)([OH2+])OP(=O)([O-])O[NH3+]</smiles>

2<smiles>CC(CCOC(C)(C)C(=O)NN)C(=O)OP(=O)([OH2+])OP(=O)([OH2+])O[NH3+]</smiles>

3

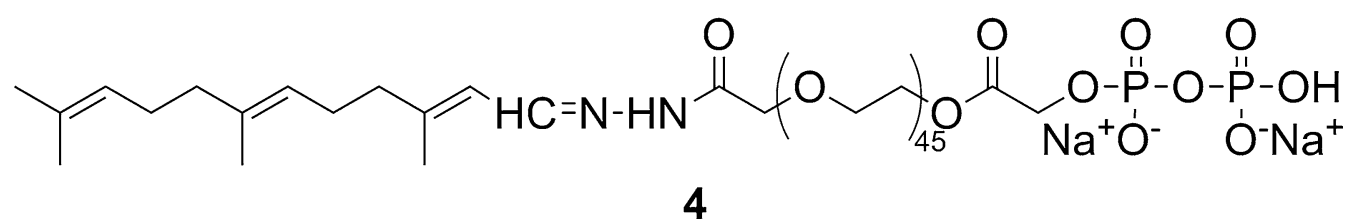

Fig. 1 Synthesis of the pH-sensitive dentotropic polymeric conjugate of PPi-PEG-hyd-Far

concentrations, and $101.63 \pm 0.04 \%$ (RSD, $0.04 \%$ ) at high concentrations. These results suggest that our HPLC method was robust and reliable. Using this method, we measured drug loading and encapsulation efficiencies to be $9.10 \pm 0.70 \%$ and $76.4 \pm 2.10 \%$ for Far-PMs, and $9.51 \pm 0.40 \%$ and $78.30 \pm 1.40 \%$ for PPi-Far-PMs.

Far was released from PPi-Far-PMs much faster at $\mathrm{pH}$ 4.5 than at pH 7.4 (Fig. 2e). At pH 4.5, nearly 90\% of Far was released within the first $30 \mathrm{~min}$, compared to only $40.60 \%$ of Far released within $24 \mathrm{~h}$ at $\mathrm{pH} 7.4$, that may depends on the $\mathrm{pH}$ sensitivity of the hydrazone bond. These results suggest that we succeeded in creating a Far delivery system that selectively and rapidly released the drug under acidic conditions.
PPi-Far-PMs bind to hydroxyapatite particles better and faster than Far-PMs

We prepared the biotechnological hydroxyapatite (Fig. 3) as a mimic of enamel in order to examine the binding of PPi-Far-PMs. PPi-Far-PMs showed much greater ability to bind hydroxyapatite than Far-PMs (Fig. 4a, b). PPiFar-PMs bound efficiently to hydroxyapatite particles within 2 min (Fig. 4c, d), which is comparable to the time most people spend brushing their teeth or rinsing their mouth with mouthwash. PPi-Far-PMs also showed greater binding rate with biotechnological hydroxyapatite than that with the commercial hydroxyapatite at each time point. 


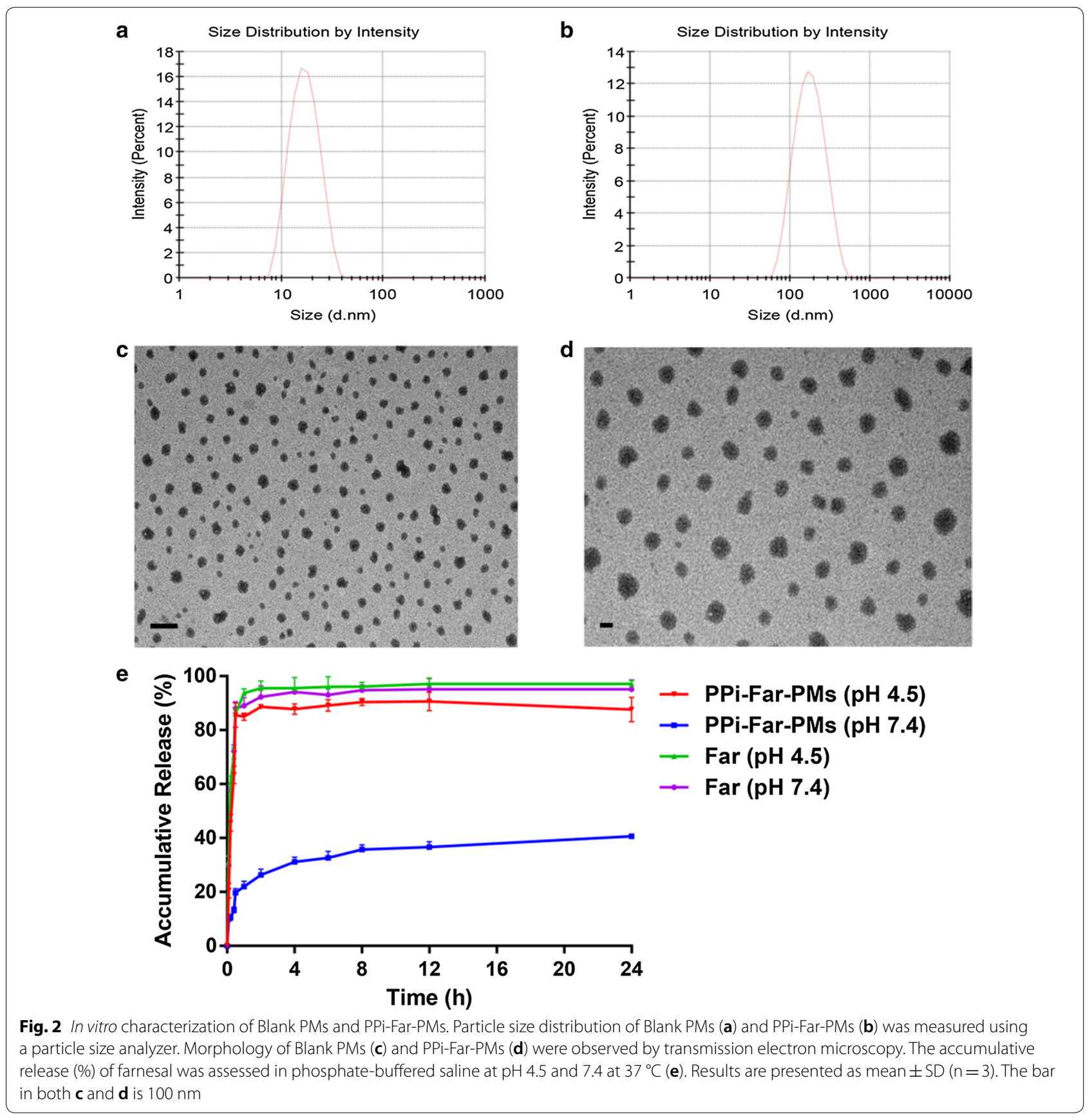

Anti-bacterial effects of Far against S. mutans

Since $S$. mutans is the main microorganism initiating caries, the MIC and MBC of Far against this microbe were measured. The bacteria appeared to be similarly susceptible to Far and farnesol based on MIC (14 vs $28 \mu \mathrm{g} / \mathrm{mL}$ ) and MBC (112 vs $112 \mu \mathrm{g} / \mathrm{mL}$; Additional file 1: Table S1) [26]. The positive control treatment with CHX showed lower MIC and MBC, but it was associated with tooth staining and calculus formation as noted previously [10].
PPi-Far-PMs enhance the anti-caries activity of Far in vivo Blank PMs and distilled water had no obvious effect on the amount of $S$. mutans in saliva from the treated rats (Fig. 5a). The other all treatments reduced the amount of $S$. mutans, while CHX and PPi-Far-PMs reduced it $(P<0.05)$ significantly. These results indicate that PPiFar-PMs can inhibit the growth of S. mutans in vivo. All animals remained in good health during the experiment and the body-weight increase was similar to that of the 

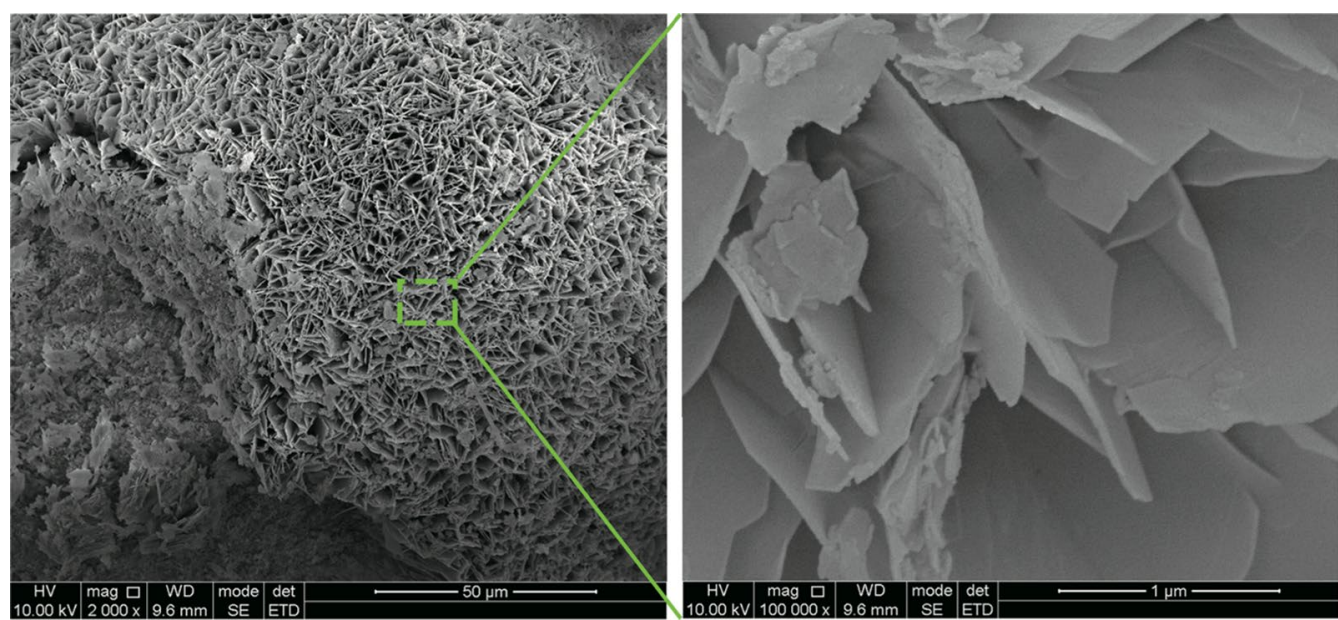

Fig. 3 Preparation of biotechnological hydroxyapatite. During preparation, the top surface of the small intestinal submucosa membrane was maintained in contact with $\mathrm{K}_{2} \mathrm{HPO}_{4}$ solution, while the lower surface was kept in contact with the $\mathrm{Ca}\left(\mathrm{CH}_{3} \mathrm{COO}\right)_{2}$ solution. After incubation for 10 days at $37^{\circ} \mathrm{C}$, the entire submucosa membrane with hydroxyapatite was peeled off and analyzed by scanning electron microscopy. The magnification in the left image is $\times 2000$, and the right image shows a zoomed view at $\times 100000$

normal rats rats (Fig. 5b). We observed that the physiological conditions of rats such as diet and defecation are normal. No local oral mucosal allergy was observed in any of the animals during the drug intervention.

Figure 6 shows examples of treated rats that showed Smo-E, Pro-E, Sul-E, Sul-Ds, Sul-Dm or Sul-Dx. The treatments differed in how much and how severely they caused sulcal-surface caries of molars (Fig. 7a); as expected, distilled water was associated with the most severe caries. Keyes' scores of smooth-surface caries were significantly higher with distilled water than with Far-PMs $(P<0.05)$, PPi-Far-PMs $(P<0.001)$, or CHX $(P<0.0001)$ (Table 1 and Fig. 7b). Farnesol reduced incidence of Smo-E by $18 \%$; Far, by $28 \%$; and ethanol, by $15 \%$. In contrast, Far-PMs, PPi-Far-PMs and $\mathrm{CHX}$ reduced incidence by, respectively, 50\%, $64 \%$ and $79 \%$.

The incidence of Sul-E was significantly higher with distilled water than with PPi-Far-PMs (27\% lower, $P<0.0001)$, or CHX (32\% lower, $P<0.0001$ ) (Fig. $7 \mathrm{c}$ ). In contrast, the incidence of Sul-E was only $10 \%$ lower with Far-PMs, 9\% lower with Far and 5\% lower with farnesol. Incidence of Sul-Ds was 35\% lower with PPi-Far-PMs and $42 \%$ lower with CHX than the control group treated with distilled water (Fig. 7d). The corresponding reductions in Sul-Dm incidence were $69 \%$ and $63 \%$ (Fig. 7e). These results suggest that Farloaded $\mathrm{pH}$-sensitive dentotropic PMs significantly improve the anti-caries effect of free Far. PPi-Far-PMs and $\mathrm{CHX}$ inhibit sulcal-surface caries to a similar extent.

\section{PPi-Far-PMs enhance the teeth mechanical strength in rat caries}

Molars mechanical strength was much higher in teeth from rats treated with PPi-Far-PMs than that in teeth from rats treated with Far, which in turn was higher than in teeth from animals treated with distilled water (Fig. 8a, d). PPi-Far-PMs exerted similar effects as CHX under these conditions.

\section{PPi-Far-PMs can restore the microarchitecture of teeth with caries}

The caries caused severe molars damage, as seen in BMD and BV/TV of molars from animals treated with distilled water or blank PMs. BMD and BV/TV of molars were significantly higher in groups of Far-PMs and PPiFar-PMs than that in group of the distilled water (Fig. 9, Additional file 1: Figure S6). The improvement in the group of PPi-Far-PMs was similar to that in the group of CHX. None of the other treatments significantly improved BMD or BV/TV relative to the distilled water. These results indicate that PPi-Far-PMs can effectively inhibit demineralization of the tooth enamel.

\section{Discussion}

We have synthesized pH-sensitive dentotropic PPi-PEGhyd-Far polymeric conjugate by linking PEG and Far via an acid-labile hydrazone bond, then modifying it with biodegradable PPi to make enamel-targeting PMs. PEG is a non-immunogenic, biocompatible water-soluble polymer often conjugated to therapeutic proteins and other drugs. Several of these conjugates have entered clinical 
a

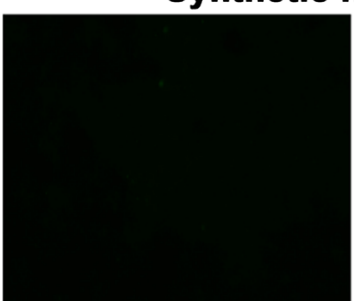

FITC

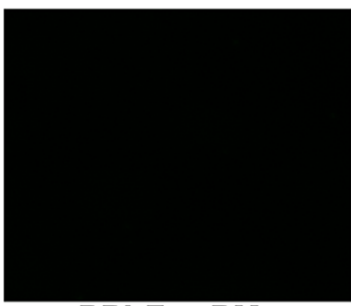

PPi-Far-PMs

c

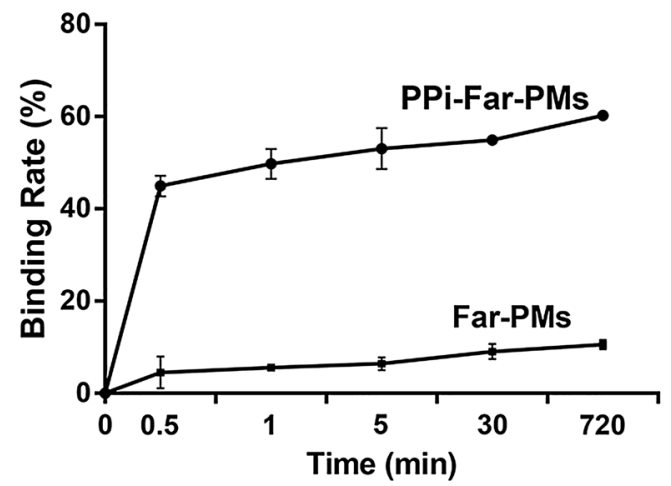

Far-PMs-FITC b Biotechnological hydroxyapatite
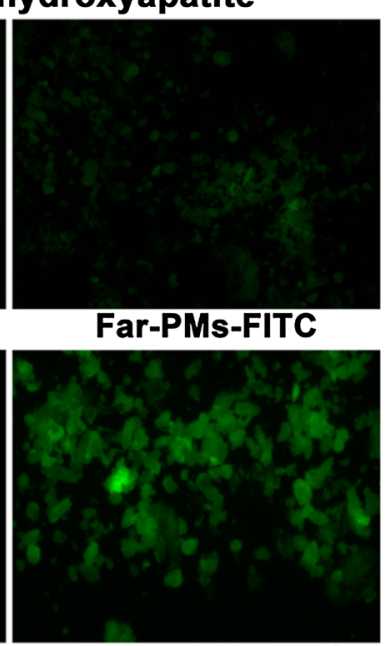

PPi-Far-PMs-FITC

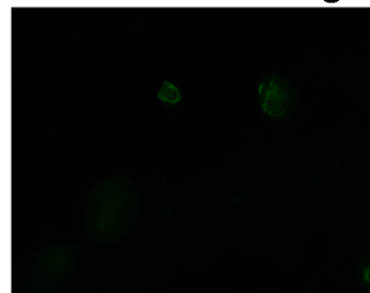

FITC

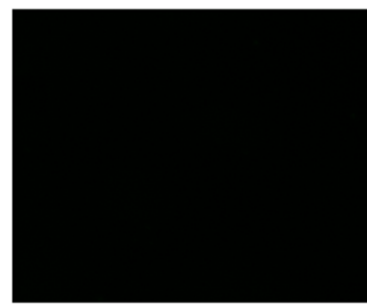

PPi-Far-PMs

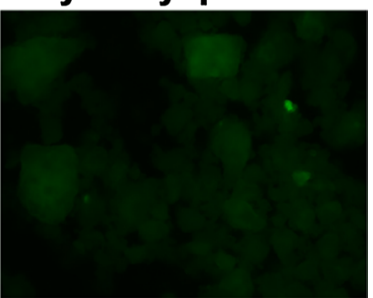

Far-PMs-FITC

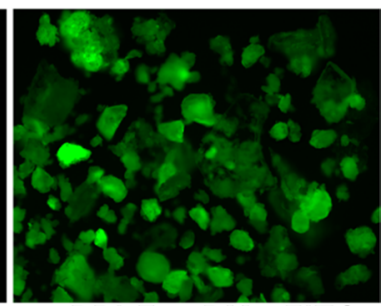

PPi-Far-PMs-FITC

d Biotechnological hydroxyapatite

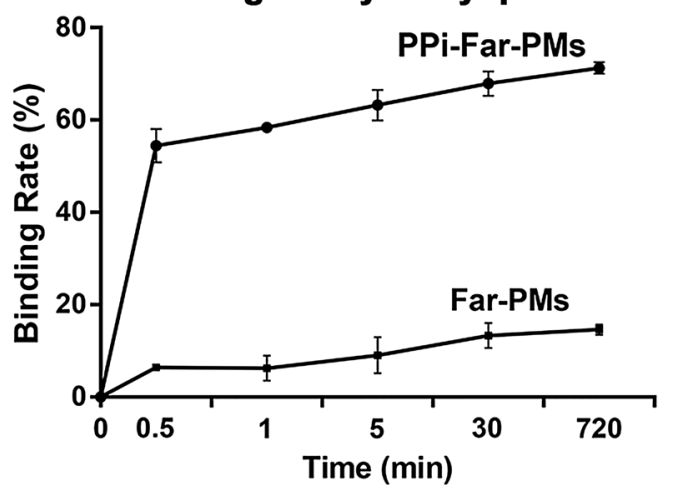

Fig. 4 The binding ability of PPi-Far-PMs to hydroxyapatite particles. PPi-Far-PMs and Far-PMs were labeled with FITC and incubated with the commercial synthetic hydroxyapatite $(\mathbf{a}, \mathbf{c})$ or with the biotechnological hydroxyapatite $(\mathbf{b}, \mathbf{d})$. The images show 30-min incubations with FITC, Far-PMs-FITC, PPi-Far-PMs or PPi-Far-PMs-FITC. Magnification, $\times 40$. Binding kinetics $(\mathbf{c}, \mathbf{d})$ were assessed by assaying the bound Far using HPLC at different time points. All data are mean \pm SD $(n=3)$

trials for cancer treatment [27-30]. The hydrazone linkage has often been used to render the release of drugs such as doxorubicin and paclitaxel selective under acidic conditions [31-34]. We prepared PPi-Far-PMs using a simple membrane hydration method that generated homogeneous, uniformly spherical micelles.

We found that Far, a derivative of the natural product farnesol, shows similar anti-bacterial efficacy as farnesol against $S$. mutans. At the same time, its incorporation into PPi-Far-PMs helps its retention in the oral cavity and led to stronger anti-bacterial and anti-caries effects than free farnesol or even Far-PMs in our rat model of induced caries. While $S$. mutans is a major component of dental plaque that causes cavities, other bacterial species also contribute, including Streptococcus sobrinus, lactobacilli and candida albicans. Future work should examine the efficacy of Far against these species in order to gain a more complete understanding of its anti-caries activity.

Consistent with the good anti-caries activity of PPi-FarPMs in our rat model, we found that the micelles bound strongly to biotechnological hydroxyapatite particles; in fact, they bound more strongly to these particles, which mimic tooth material, than to chemical hydroxyapatite. PPi-Far-PMs bound within a few minutes to hydroxyapatite and persisted for up to $12 \mathrm{~h}$, which suggests they can be effective during routine use.

We assayed the anti-caries activity of PPi-Far-PMs in a rat model of caries induced by the combination of S. mutans UA159 infection and a cariogenic diet [35, 
a

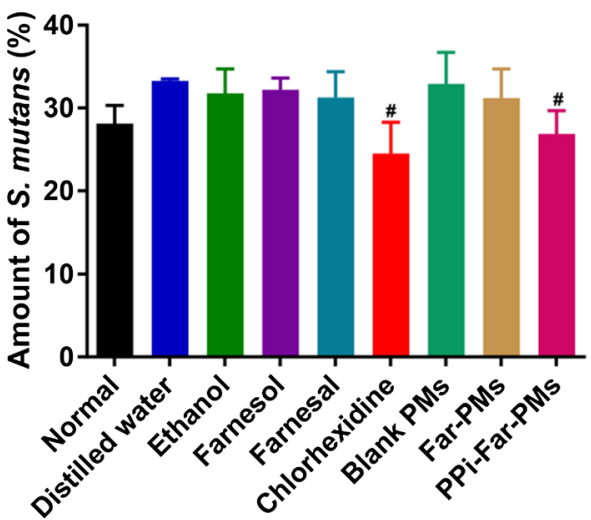

b

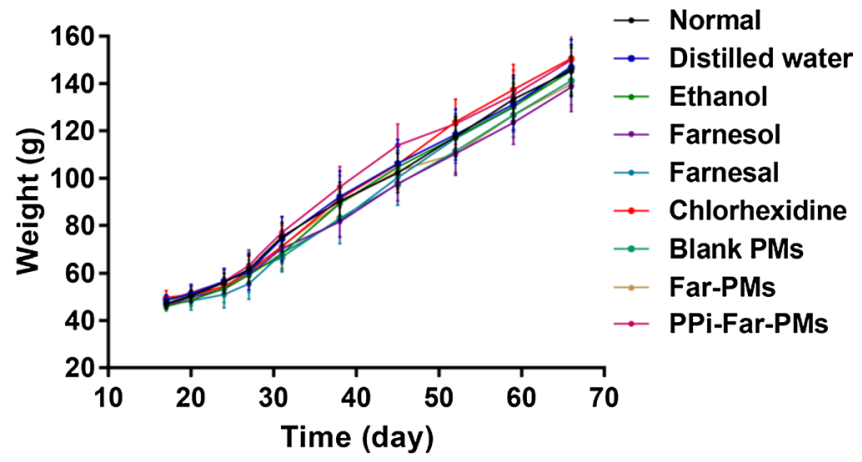

Fig. 5 Measurement of the relative levels of $S$. mutans (a) and body weight of rats (b) in the dental caries model after treatment with distilled water, $15 \%$ ethanol, farnesol, farnesal, chlorhexidine, blank PMs, Far-PMs and PPi-Far-PMs, respectively. Values are expressed as mean \pm standard deviation. ${ }^{\#} P<0.05$ vs the control group treated with distilled water
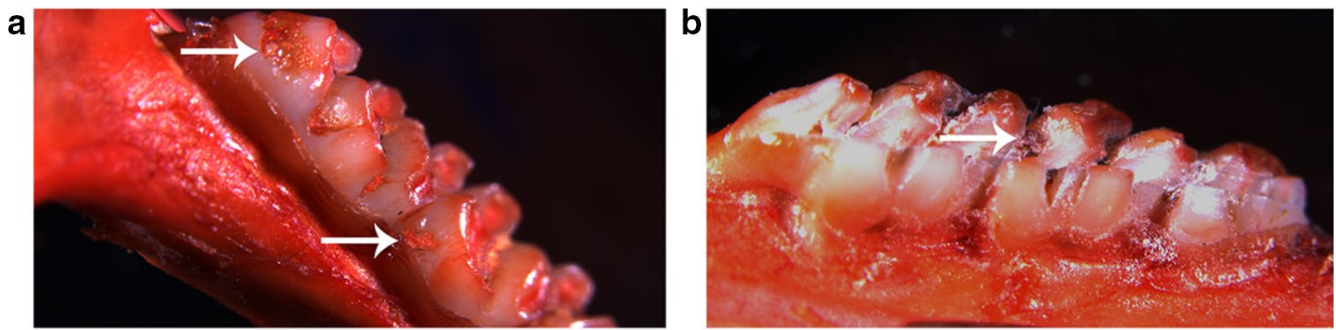

C
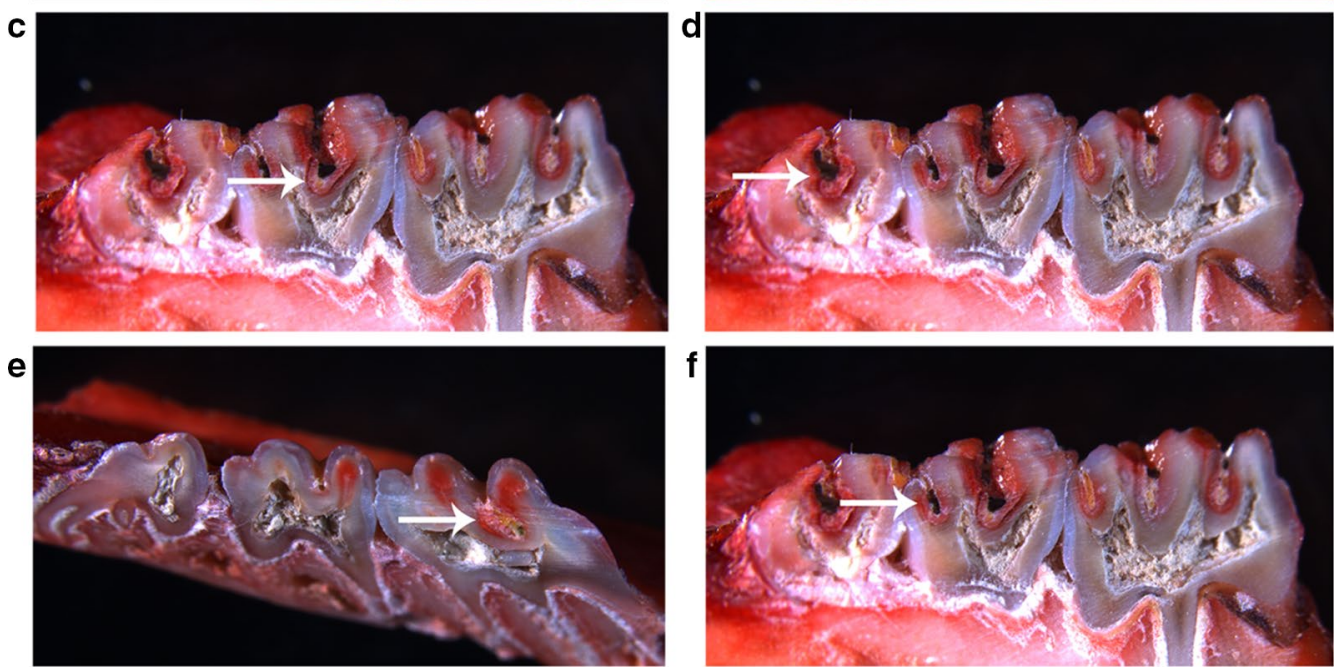

Fig. 6 Representative stereo-micrographs of molars from the rat model of caries, showing smooth-surface caries with enamel affected (a),

proximal-surface with enamel affected (b), sulcal-surface with enamel affected (c), sulcal-surface with dentin exposed (d), sulcal-surface with 3/4 of the dentin affected (e), and sulcal-surface with whole dentin affected (f). Bold arrows show examples of the indicated damage. Magnification, 14x

36]. PPi-Far-PMs significantly reduced the incidence and severity of smooth and sulcal surface caries compared with the control group, while free Far and farnesol showed no obvious difference from controls. Mechanical tests and micro-CT of molars showed that PPi-Far-PMs effectively protected teeth from damage and bone loss. Meanwhile, we also found that when the PPi-Far-PMs drug-loading concentration was at low-dose of $0.55 \mathrm{mg} /$ $\mathrm{mL}$, Far can reduce the incidence of Smo-E by $51 \%$, and that of Sul-E by $17 \%$, respectively. In compressive 


\section{(See figure on next page.)}

Fig. 7 Treatments effects on the development of dental caries in rats. Animals were treated with distilled water, 15\% ethanol, farnesol, farnesal, chlorhexidine, blank PMs, Far-PMs and PPi-Far-PMs, respectively. a The smooth-surface and sulcal-surface caries of molars were observed under a stereomicroscope $(\times 14)$. The white arrow indicated the smooth-surface caries while the green arrow indicating the sulcal-surface caries. Quantitative assessment was based on carious lesion severity of smooth-surface and sulcal-surface according to the Keyes'scoring system, including $\mathbf{b}$ the smooth-surface with enamel affected (Smo-E), $\mathbf{c}$ sulcal-surface with enamel affected (Sul-E), $\mathbf{d}$ sulcal-surface with dentin exposed (Sul-Ds), e sulcal-surface with $3 / 4$ of the dentin affected (Sul-Dm). Values are expressed as mean \pm standard deviation $(n=7)$. The Tamhane's T2 test $\left({ }^{*} P<0.05,{ }^{* *} P<0.01,{ }^{* * *} P<0.0001\right)$ was used to assess the treatment efficacy. Symbols represented statistical significance of the labeled groups relative to the group treated with distilled water $\left(\# P<0.05,{ }^{\# \#} P<0.01,{ }^{\# \#} P<0.001,{ }^{\# \# \# P} P<0.0001\right)$. The caries reduction rate was determined as described in Methods

strength experiments, resulted from the low-dose group of PPi-Far-PMs, values of compressive strength, compressive strength, compressive yield stress and maximum stress were $172.49 \pm 5.89 \mathrm{MPa}, 20.50 \pm 2.33 \mathrm{MPa}$, $10.78 \pm 1.65 \mathrm{MPa}$, and $15.47 \pm 1.90 \mathrm{MPa}$, respectively; while in distilled water group, they were $127.77 \pm 6.45 \mathrm{MPa}, 13.47 \pm 1.32 \mathrm{MPa}, 4.32 \pm 1.72 \mathrm{MPa}$, and $7.17 \pm 1.34 \mathrm{MPa}$, respectively. Statistical comparisons indicated that the low-dose of PPi-Far-PMs treatments showed remarkable differences compared to distilled water $(P<0.0001, P<0.05, P<0.01, P<0.01)$. Meanwhile, we found that BMD and BV/TV of molars were significantly higher with the low-dose PPi-Far-PMs (BMD 0.694 $\pm 0.041 \mathrm{~g} / \mathrm{cm}^{3}$, BV/TV $0.505 \pm 0.005$ ) than with distilled water (BMD $0.531 \pm 0.045 \mathrm{~g} / \mathrm{cm}^{3}, \mathrm{BV} / \mathrm{TV}$ $0.362 \pm 0.010 ; P<0.01, P<0.0001)$. This reminds us that the drug-delivery system of PPi-Far-PMs can reduce the dosage of the Far and prevent effectively the dental caries. We attribute these results to the ability of PPi-FarPMs killing bacteria in the acidic microenvironment of dental plaques and binding efficiently to hydroxyapatite, and thereby inhibited demineralization and promoted re-mineralization.

\section{Conclusions}

In this study, we developed the drug-delivery system of PPi-Far-PMs that binds efficiently and rapidly to hydroxyapatite, and that releases Far selectively in the acidic microenvironment of dental plaque. We showed in vitro that Far on its own has similar anti-bacterial activity as its parent farnesol against cariogenic $S$. mutans UA159. We further showed that incorporating Far into PPi-Far-PMs made it much more effective than free farnesol at treating dental caries in a rat model. This suggests that PMs can improve the water solubility and prolong the retention time in the oral cavity. This novel $\mathrm{pH}$-sensitive drug delivery system shows potential for targeted anti-bacterial treatment against dental caries, and it may be useful for delivering other agents to treat disease in the oral cavity.

\section{Materials and methods Materials}

Far, farnesol, and TBAP were supplied by Sigma-Aldrich (St. Louis, MO, USA). Chlorhexidine digluconate (CHX) was obtained from Macklin (Shanghai, China). HO$\mathrm{PEG}_{2000}-\mathrm{NHNH}_{2}$ was obtained from Xi'an Ruixi Biological Technology (Xi'an, China), and $\mathrm{mPEG}_{2000}-\mathrm{PLA}_{2000}$ was purchased from Jinan Daigang Biomaterials (Jinan, China). Mitis Salivarius agar was purchased from BD Biosciences (San Jose, CA, USA). Brain heart infusion broth and agar were purchased from Hopebio (Qingdao, China). S. mutans UA159 (ATCC 700610) was purchased from the China Center of Industrial Culture Collection (Beijing, China).

\section{Synthesis of $\mathrm{pH}$-sensitive dentotropic polymeric conjugate} The $\mathrm{pH}$-sensitive dentotropic polymeric conjugate PPiPEG-hyd-Far was synthesized as shown in Fig. 1. The commercially available $\mathrm{HO}-\mathrm{PEG}_{2000}-\mathrm{NHNH}_{2}$ was protected with a $t$-butyloxy carbonyl (Boc) group, and then subjected to esterification and bromination to give PPiPEG-NHNH-Boc. The Boc group was removed and subsequent hydrazide reaction generated PPi-PEG-hyd-Far, whose identity was confirmed using ${ }^{1} \mathrm{H}$ and ${ }^{13} \mathrm{C}$ NMR. The specific reaction conditions for each compound are detailed below.

\section{Synthesis of compound 1: tert-butyl 2-(2-(2-hydroxyeth- oxy)acetyl)hydrazine-1-carboxylate (HO-PEG-NHNH-Boc)} Triethylamine (25.3 $\mathrm{mg}, 0.25 \mathrm{mmol}$ ) was added to a stirred solution of $\mathrm{HO}-\mathrm{PEG}_{2000}-\mathrm{NHNH}_{2}(500 \mathrm{mg}$, $0.25 \mathrm{mmol}$ ) in methanol. The mixture was cooled to $0{ }^{\circ} \mathrm{C}$ and di-tert-butyl dicarbonate $(65.5 \mathrm{mg}, 0.30 \mathrm{mmol})$ was added slowly to the reaction solution. The reaction mixture was stirred at $0{ }^{\circ} \mathrm{C}$ for $1 \mathrm{~h}$, then warmed to room temperature and stirred for $24 \mathrm{~h}$. The solution was concentrated under vacuum, and the product was precipitated in anhydrous ether three times, dried overnight under vacuum and stored at $-20{ }^{\circ} \mathrm{C}$ for an overall yield of $72.4 \%$. 

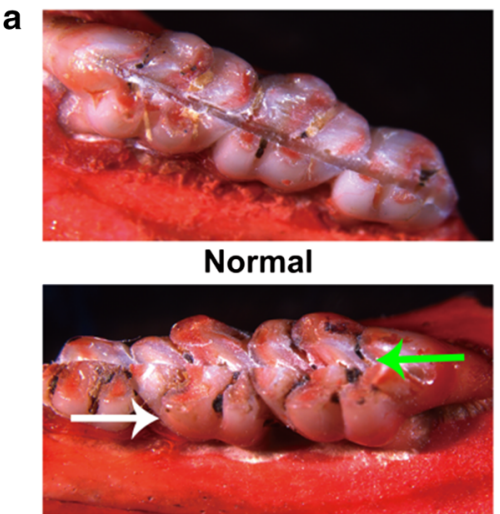

Farnesol

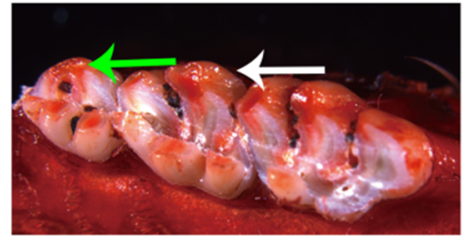

Blank PMs

b

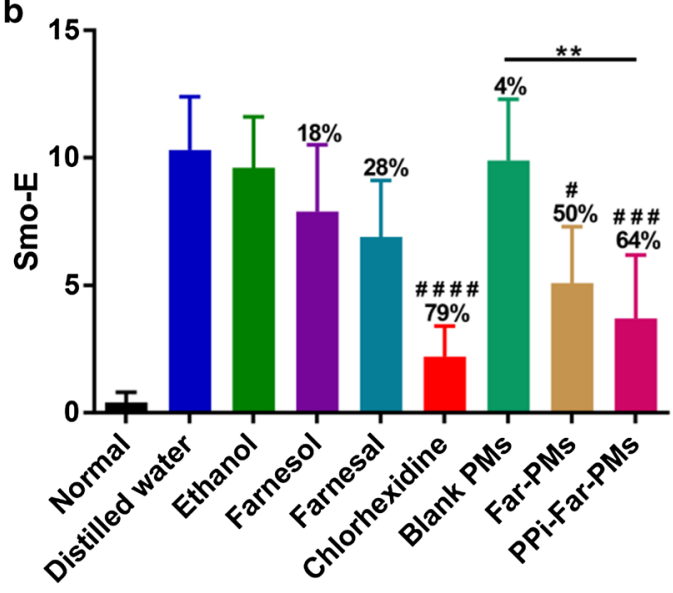

d

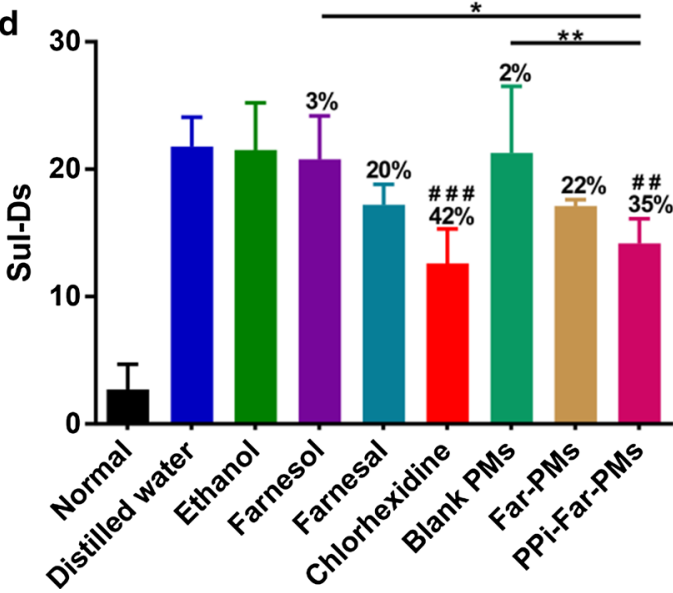

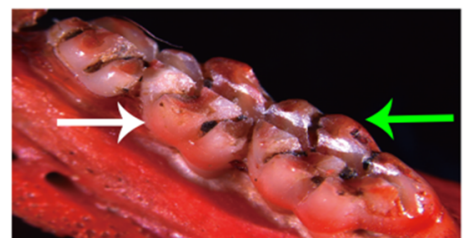

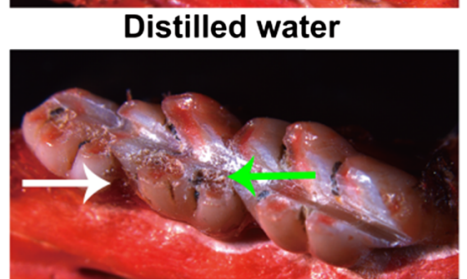

Farnesal

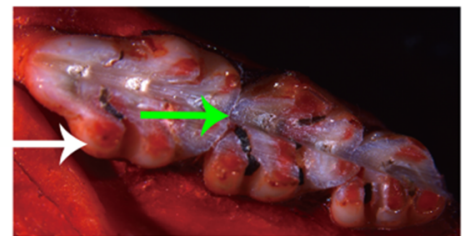

Far-PMs

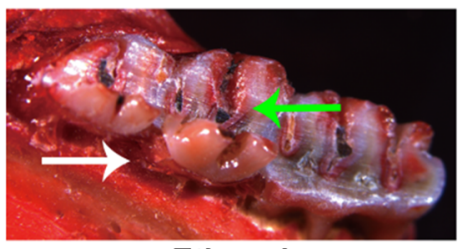

Ethanol

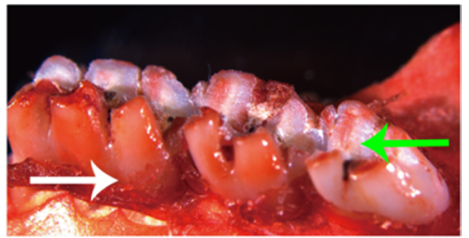

Chlorhexidine

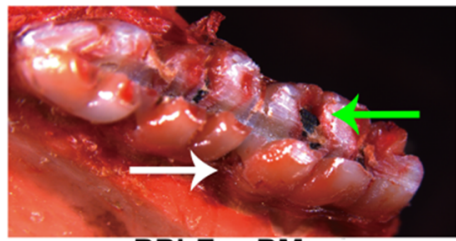

PPi-Far-PMs
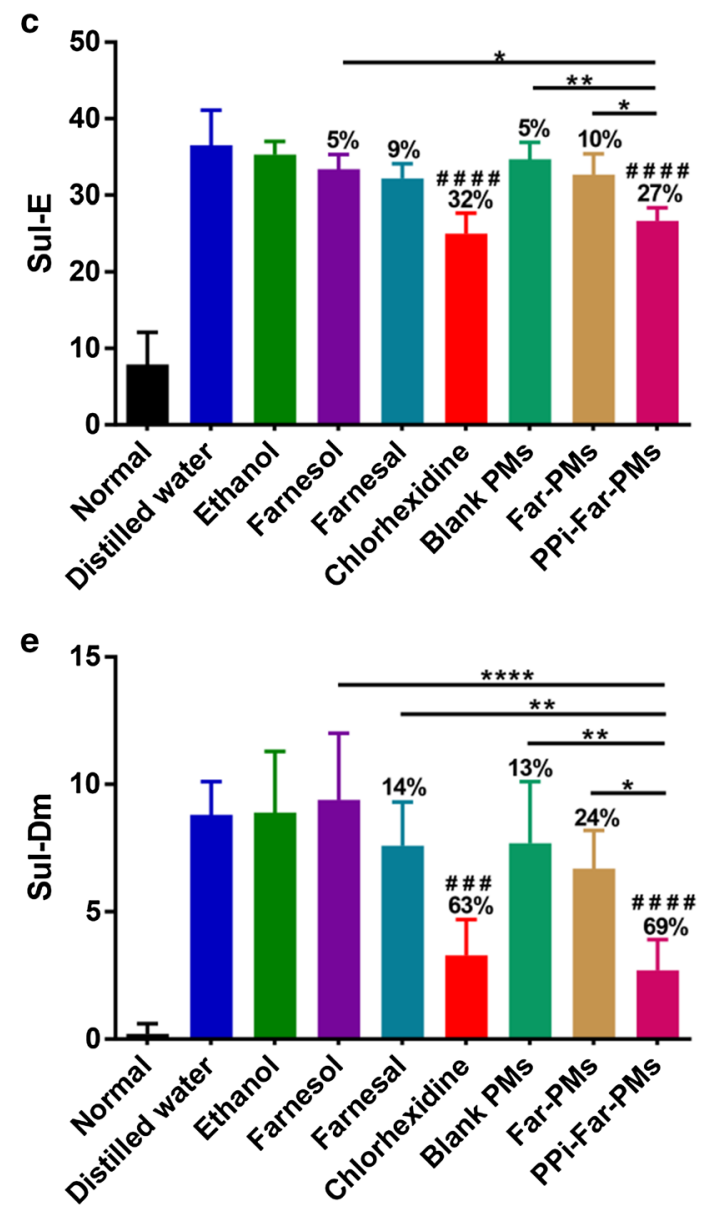
Table 1 Keyes' scores for assessment the efficiency of different treatments on the development of dental caries in rats

\begin{tabular}{|c|c|c|c|c|c|}
\hline \multirow[t]{2}{*}{ Treatment } & \multirow{2}{*}{$\begin{array}{l}\text { Smooth- } \\
\text { surface } \\
\text { caries } \\
\text { E }\end{array}$} & \multicolumn{4}{|c|}{ Sulcal-surface caries } \\
\hline & & $E$ & Ds & $\mathrm{Dm}$ & Dx \\
\hline $\begin{array}{c}\text { Distilled } \\
\text { water }\end{array}$ & $10.3(2.1)^{\mathrm{a}}$ & $36.5(4.6)^{\mathrm{a}}$ & $21.8(2.3)^{\mathrm{a}}$ & $8.8(1.3)^{\mathrm{a}}$ & $0.7(0.8)^{\mathrm{a}}$ \\
\hline $\begin{array}{l}\text { 15\% Etha- } \\
\text { nol }\end{array}$ & $9.6(2.0)^{\mathrm{a}}$ & $35.3(1.7)^{\mathrm{ac}}$ & $21.5(3.7)^{\mathrm{a}}$ & $8.9(2.4)^{\mathrm{a}}$ & $0.5(0.5)^{\mathrm{a}}$ \\
\hline Farnesol & $7.9(2.6)^{\mathrm{ac}}$ & $33.4(1.9)^{\mathrm{ac}}$ & $20.8(3.4)^{a}$ & $9.4(2.6)^{\mathrm{a}}$ & $0.5(0.7)^{\mathrm{a}}$ \\
\hline Farnesal & $6.9(2.2)^{\mathrm{ac}}$ & $32.2(1.9)^{\mathrm{acd}}$ & $17.2(1.6)^{\mathrm{ac}}$ & $7.6(1.7)^{\mathrm{a}}$ & n.d. \\
\hline $\begin{array}{l}\text { Chlorhex- } \\
\text { idine }\end{array}$ & $2.2(1.2)^{\mathrm{bd}}$ & $25(2.7)^{b}$ & $12.6(2.7)^{b c}$ & $3.3(1.4)^{\text {bcde }}$ & n.d. \\
\hline Blank PMs & $9.9(2.4)^{\mathrm{a}}$ & $34.7(2.2)^{\mathrm{ac}}$ & $21.3(5.2)^{\mathrm{a}}$ & $7.7(2.4)^{\mathrm{a}}$ & $0.4(0.4)^{\mathrm{a}}$ \\
\hline Far-PMs & $5.1(2.2)^{\mathrm{bc}}$ & $32.7(2.7)^{\mathrm{ac}}$ & $17.1(0.5)^{\mathrm{ac}}$ & $6.7(1.5)^{\mathrm{ac}}$ & n.d. \\
\hline PPi-Far-PMs & $3.7(2.5)^{\mathrm{bcd}}$ & $26.7(1.7)^{\mathrm{bd}}$ & $14.2(1.9)^{\mathrm{bd}}$ & $2.7(1.2)^{\text {bde }}$ & n.d. \\
\hline
\end{tabular}

The Keyes' scores are presented as the average of all the measurements $(n=7)$. Values followed by the same superscripts $(a, b, c, d, e)$ are not significantly different from each other $(P>0.05$; comparison for all pairs using Tamhane's $T 2$ test)

E, enamel affected; Ds, dentin exposed; Dm, 3/4 of the dentin affected; and Dx, whole dentin affected; n.d., not detectable

\section{Synthesis of compound 2: sodium}

2,2-dimethyl-4,7,13-trioxo-3,9,12-trioxa-5,6-diazatetradecan-14-yl hydrogen diphosphate (PPi-PEG-NHNH-Boc)

Bromoacetic acid (43.4 mg, $0.31 \mathrm{mmol}$ ) was added to a stirred solution of compound 1 (500 $\mathrm{mg}, 0.24 \mathrm{mmol}$ ) in anhydrous dichloromethane (DCM). The solution was cooled to $0{ }^{\circ} \mathrm{C}$, then 4-dimethylaminopyridine (DMAP, $2.9 \mathrm{mg}, 0.024 \mathrm{mmol}$ ) and $\mathrm{N}, \mathrm{N}^{\prime}$-dicyclohexylcarbodiimide (DCC, $54.4 \mathrm{mg}, 0.26 \mathrm{mmol}$ ) were added slowly, and the mixture was stirred at room temperature for $24 \mathrm{~h}$. The reaction mixture was filtered and concentrated under vacuum, and the product was precipitated in anhydrous ether. The precipitate was filtered and dialyzed against water for $24 \mathrm{~h}$. TBAP (415.1 $\mathrm{mg}, 0.46 \mathrm{mmol}$ ), previously dissolved in anhydrous acetonitrile $\left(\mathrm{CH}_{3} \mathrm{CN}\right)$, was added slowly to the dialyzed product, and the mixture was stirred at room temperature for $12 \mathrm{~h}$. The solution was concentrated under vacuum, and the product was precipitated into anhydrous ether. The product was filtered, dialyzed against $\mathrm{NaCl}$ solution and then dialyzed against water at $0{ }^{\circ} \mathrm{C}$ for $10 \mathrm{~h}$. The purified product was freezedried and stored at $-20{ }^{\circ} \mathrm{C}$ for overall yield of $80.7 \%$.

Synthesis of compound 3: sodium 2-(2-(2-hydrazinyl-2-oxoethoxy)ethoxy)-2-oxoethyl hydrogen diphosphate (PPi-PEG-NHNH ${ }_{2}$ )

Compound 2 (500 mg, $0.18 \mathrm{mmol}$ ) was de-protected using zinc bromide $\left(\mathrm{ZnBr}_{2}, 81 \mathrm{mg}, 0.36 \mathrm{mmol}\right)$ in dichloromethane (DCM) for $4 \mathrm{~h}$. The solution was filtered and concentrated under vacuum, and the product was precipitated in anhydrous ether three times and against water at $0{ }^{\circ} \mathrm{C}$ for $10 \mathrm{~h}$. Then the purified product was dried overnight under vacuum and stored at $-20{ }^{\circ} \mathrm{C}$ with overall yield of $80.20 \%$.

Synthesis of compound 4: sodium 2-oxo-2-(2-(2-oxo-2-(2 -((2E,6E)-3,7,11-trimethyldodeca-2,6,10-trien-1-ylidene) hydrazinyl)ethoxy)ethoxy)ethyl hydrogen diphosphate (PPi-PEG-hyd-Far)

Compound 3 (500 mg, $0.19 \mathrm{mmol}$ ) and Far $(61.0 \mathrm{mg}$, $0.28 \mathrm{mmol}$ ) were dissolved in anhydrous methanol, and acetic acid (1.1 mg, $0.02 \mathrm{mmol}$ ) was added to the reaction solution. After stirring at room temperature for $48 \mathrm{~h}$, the solution was concentrated under vacuum, and the product was precipitated in anhydrous ether three times. Then the purified product was dried overnight under vacuum and stored at $-20{ }^{\circ} \mathrm{C}$ with overall yield of $79.80 \%$.

\section{Preparation of polymeric micelles}

The membrane hydration method [37] was used to prepare blank polymeric micelles (PMs), Far-loaded pH-sensitive polymeric micelles (Far-PMs), as well as PPi-targeted and Far-loaded pH-sensitive dentotropic polymeric micelles (PPi-Far-PMs). The following components were used: $\mathrm{mPEG}_{2000}-\mathrm{PLA}_{2000}$ (A), PEG-hydFar (B), PPi-PEG-hyd-Far (C), and Far (D). The weight ratios of components $\mathrm{A} / \mathrm{B} / \mathrm{C} / \mathrm{D}$ were optimized using the central composite design $[38,39]$ to be as following (Table 2): blank PMs, 40/0/0/0; Far-PMs, 20/20/0/4; and PPi-Far-PMs, 20/0/20/4. The components were dissolved in $4.0 \mathrm{~mL}$ of acetonitrile in a round-bottom flask, then the acetonitrile was evaporated under vacuum at $55{ }^{\circ} \mathrm{C}$ to obtain a thin film. Residual acetonitrile was completely removed under vacuum overnight at room temperature. The dried thin film was hydrated with ultra-pure water $(2.0 \mathrm{~mL})$ and sonicated for $3 \mathrm{~min}$ in a bath sonicator, stirred for $12 \mathrm{~h}$ at room temperature and filtered through a $0.22-\mu \mathrm{m}$ membrane. The micellar solution was freezedried and stored at $4{ }^{\circ} \mathrm{C}$.

The same method and the additional reagent FITCPEG (E) were used to prepare FITC-labeled PMs in the following weight ratios: Far-PMs-FITC, A/B/D/E: 20/20/4/1; and PPi-Far-PMs-FITC, A/C/D/E: 20/20/4/1.

\section{Characterization of polymeric micelles}

The size and zeta potential of polymeric micelles were measured using dynamic light scattering (Zetasizer Nano ZS90, Malvern Instruments, Malvern, UK). Morphology of blank PMs and PPi-Far-PMs were observed using transmission electron microscopy (JEM-100SX, Japan). Critical micelle concentration (CMC) was determined 

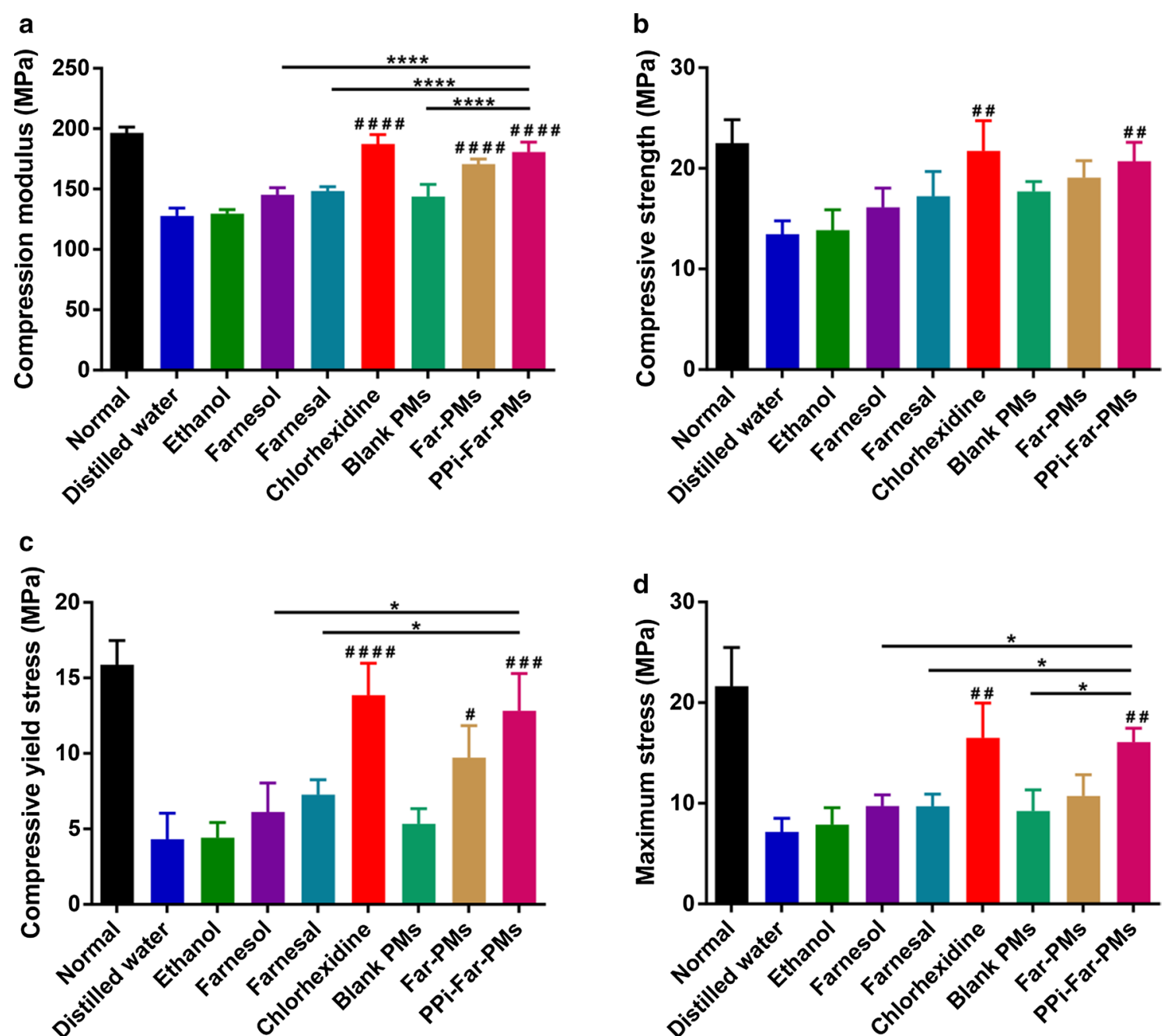

Fig. 8 Effects of different treatments on the biomechanical properties of molars including the compression modulus (a), compressive strength (b), compressive yield stress (c) and maximum stress (d). Rats were treated with distilled water, 15\% ethanol, farnesol, farnesal, chlorhexidine, blank PMs, Far-PMs and PPi-Far-PMs, respectively. At 5 weeks after administration, the molars were subjected to the mechanical compressive strength testing via a biomechanical testing system. Values are expressed as mean \pm SD. The Tamhane's T2 test test $\left({ }^{*} P<0.05,{ }^{* * *} P<0.0001\right)$ was used to assess the treatment efficacy. Symbols represented statistical significance of the labeled groups with the group treated with distilled water $\left(\# P<0.05\right.$, ${ }^{\#} P<0.01$, $\#$ \#\# $<$ 0.001, \#\#\#\# $<$ 0.0001)

using fluorescence spectroscopy and pyrene as the hydrophobic fluorescent probe.

Encapsulation and drug-loading efficiencies were calculated using the ultrafiltration method and an ultrafiltration column with a molecular weight cut-off of $3 \mathrm{kDa}$ (Solarbio Science and Technology, Beijing, China). Far was quantified on an Agilent ZORBAXSB-C18 column $(4.6 \times 150 \mathrm{~mm}, 5 \mu \mathrm{m})$ at $25{ }^{\circ} \mathrm{C}$ attached to an Agilent 1260 HPLC system (Infinity, USA). The mobile phase was acetonitrile and ultrapure water $(80: 20, v / v)$ and the flow rate was $1 \mathrm{~mL} / \mathrm{min}$. The detection wavelength was $216 \mathrm{~nm}$. Encapsulation efficiency was calculated using the equation:

\section{Encapsulation efficiency (\%) \\ $=($ Weight of Far in micelles $) /$ \\ (Weight of total Far) $\times 100 \%$}

Drug-loading efficiency was calculated using the equation:

$$
\begin{aligned}
& \text { Drug - loading efficiency }(\%) \\
& =\text { (Weight of Far in micelles) } / \\
& \quad(\text { Weight of total micelles }) \times 100 \% .
\end{aligned}
$$

Release of Far from PPi-Far-PMs was investigated in vitro using the dialysis method. Briefly, $1 \mathrm{~mL}$ of 
a

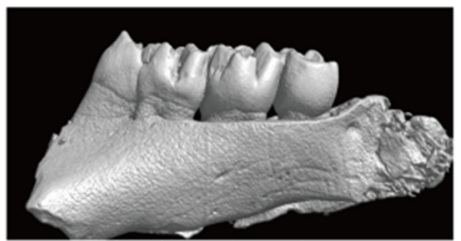

Distilled water

b

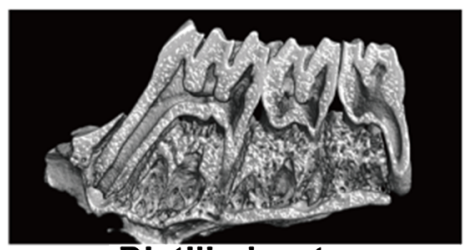

Distilled water

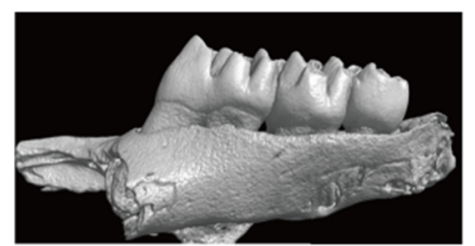

Chlorhexidine

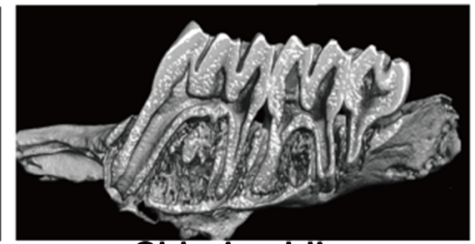

Chlorhexidine

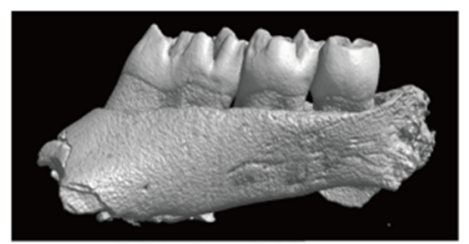

PPi-Far-PMs

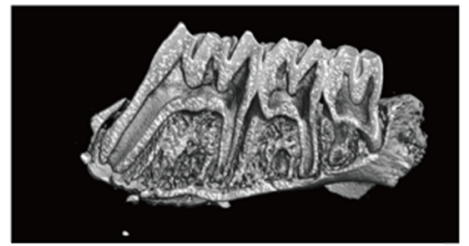

PPi-Far-PMs
C

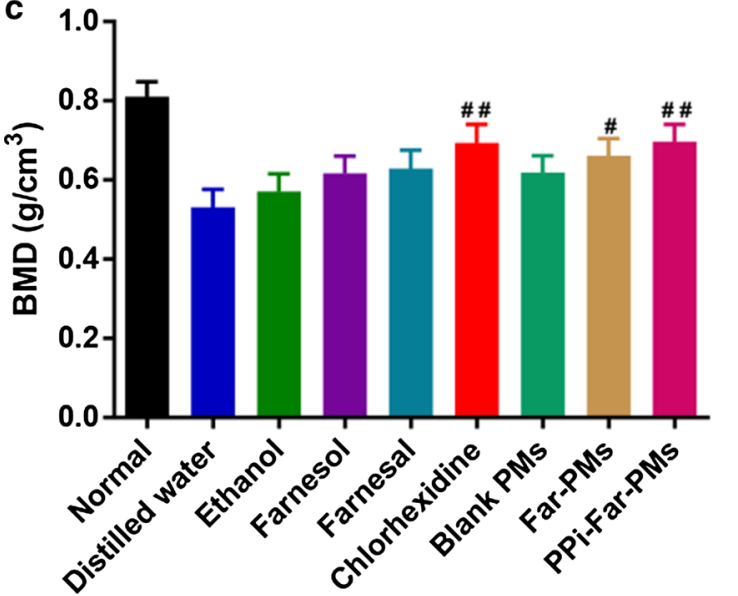

d

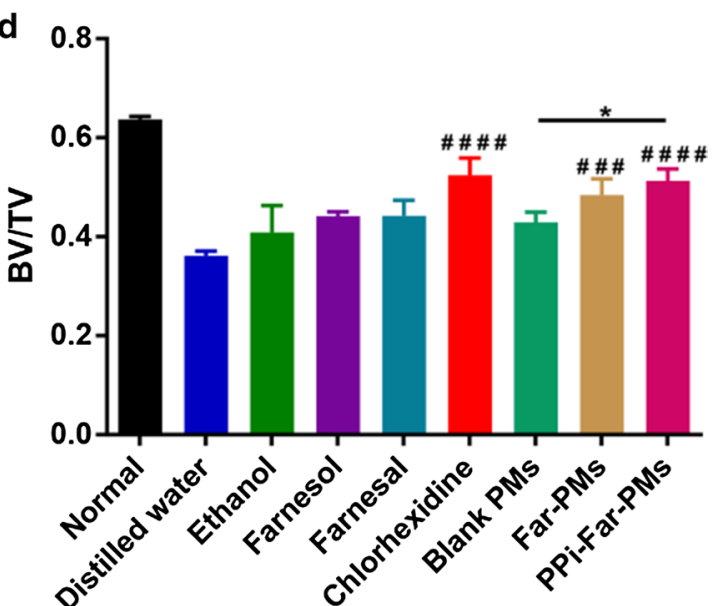

Fig. 9 Effects of different treatments on the microarchitecture of molars in rats. Animals were treated with distilled water, 15\% ethanol, farnesol, farnesal, chlorhexidine, blank PMs, Far-PMs and PPi-Far-PMs, respectively. After 5 weeks, the molars of rats were analyzed using high-resolution micro-computed tomography to obtain three-dimensional reconstruction pictures of the smooth-surface molars (a) and sulcal-surface molars (b). The bone mineral density (BMD, c) and bone volume per tissue volume (BV/TV, D) were quantitatively measured. Data were expressed as mean $\pm S D(n=7)$. The Tamhane's T2 test $\left({ }^{*} P<0.05\right)$ was used to assess for treatment efficacy. Symbols represented statistical significance of the labeled groups with the group treated with distilled water $(\# P<0.05, \# P<0.01$, \#\#\# $P<0.001$, \#\#\# $P<0.0001)$

PPi-Far-PMs or free Far were placed into separate dialysis bags with a molecular weight cut-off of $1 \mathrm{kDa}$ (Spectrumlabs, USA) and dialyzed at $37^{\circ} \mathrm{C}$ against phosphate-buffered saline (PBS) at $\mathrm{pH} 4.5$ and 7.4 with gentle stirring. At different time points $(0.1,0.2,0.4,0.5,1,2,4,6,8,12$ and $24 \mathrm{~h}), 0.5 \mathrm{~mL}$ of release medium was removed and replaced with fresh medium. Far was quantified by HPLC as described above, and experiments were performed three times.

\section{Binding of PPi-Far-PMs to hydroxyapatite particles in vitro} As we all know, the most important component of the tooth enamel is hydroxyapatite [24]. To mimic the binding process of PPi-Far-PMs with tooth enamel, we used a small intestinal submucosa as the bio-mineralization template to prepare plate-like, single-crystal hydroxyapatite, referred to henceforth as 'biotechnological hydroxyapatite'. Meanwhile, we used commercially available hydroxyapatite (Macklin, Shanghai, China) as the control.

\section{Synthesis and characterization of hydroxyapatite}

Small intestinal submucosa was prepared as described [40]. The reaction device included a beaker and a centrifuge tube with a hole in the middle of the cap. The submucosa membrane covered the hole to seal the centrifuge tube, which was filled with a solution of $\mathrm{K}_{2} \mathrm{HPO}_{4}(30 \mathrm{~mL}$, $0.1 \mathrm{M})$. This tube was inverted and soaked into the beaker filled with a solution of $\mathrm{Ca}\left(\mathrm{CH}_{3} \mathrm{COO}\right)_{2}(30 \mathrm{~mL}, 0.1 \mathrm{M})$. This reaction system, which mimics bone mineralization 
Table 2 Composition and characterization of polymeric micelles

\begin{tabular}{lllllll}
\hline Polymeric micelles & $\begin{array}{l}\text { Components } \\
\text { (weight ratio, } \\
\text { A/B/C/D) }\end{array}$ & Particle size $(\mathbf{n m})$ & $\begin{array}{l}\text { Polydispersity } \\
\text { index }\end{array}$ & $\begin{array}{l}\text { Zeta potential } \\
\text { (mV) }\end{array}$ & $\begin{array}{l}\text { Drug loading } \\
\text { efficiency }\end{array}$ & $\begin{array}{l}\text { Encapsulation } \\
\text { efficiency }\end{array}$ \\
\hline Blank PMs & $40 / 0 / 0 / 0$ & $15.95 \pm 0.10$ & $0.086 \pm 0.008$ & $-0.71 \pm 0.07$ & - & - \\
Far-PMs & $20 / 20 / 0 / 4$ & $136.17 \pm 0.49$ & $0.268 \pm 0.006$ & $-2.72 \pm 0.14$ & $9.10 \pm 0.70 \%$ & $76.40 \pm 2.10 \%$ \\
PPi-Far-PMs & 20/0/20/4 & $146.20 \pm 0.87$ & $0.234 \pm 0.012$ & $-4.92 \pm 0.24$ & $9.51 \pm 0.40 \%$ & $78.30 \pm 1.40 \%$ \\
\hline
\end{tabular}

A, $\mathrm{mPEG}_{2000}-\mathrm{PLA} \mathrm{A}_{2000} ; \mathrm{B}, \mathrm{PEG}-\mathrm{hyd}$-Far; C, PPi-PEG-hyd-Far; D, Farnesal. Values are mean $\pm \mathrm{SD}$ from three independent experiments

conditions, was incubated at $37{ }^{\circ} \mathrm{C}$ for 10 days. Care was taken to ensure that the top and bottom surfaces of the submucosa membrane remained in contact with liquid [41]. Morphology of the biotechnological hydroxyapatite was analyzed using scanning electron microscopy (Inspect F50, FEI, America).

\section{Binding potential and kinetics of PPi-Far-PMs on hydroxyapatite}

Solutions of FITC-labeled PPi-Far-PMs and Far-PMs were mixed with biotechnological or commercial hydroxyapatite in round-bottom flasks (at $\mathrm{pH} 7.4$ ). After incubation with gentle stirring for $30 \mathrm{~min}$ at room temperature, the mixture was filtered, washed with PBS three times, and freeze-dried. Finally, the hydroxyapatite was observed under a fluorescence microscope (FL, AMG, America).

For analysis of binding kinetics, PPi-Far-PMs or FarPMs $(1 \mathrm{~mL})$ were mixed with either kind of hydroxyapatite particles $(50 \mathrm{mg})$ and incubated at room temperature. At certain time points $(0,0.5,1,5,30$ and $720 \mathrm{~min})$, hydroxyapatite was removed by centrifugation $(10,000 \mathrm{~g}$, $5 \mathrm{~min}$ ) and the supernatant was collected. The amount of Far in the micelle supernatant after binding $\left(\mathrm{W}_{\text {left }}\right)$ was analyzed by HPLC as described above. The binding rate (\%) at each time point was calculated as follows: Binding rate $(\%)=\left(\mathrm{W}_{\text {total }}-\mathrm{W}_{\text {left }}\right) / \mathrm{W}_{\text {total }} \times 100 \%$.

\section{Anti-bacterial activity of Far}

The ability of Far to kill S. mutans UA159, a proven virulent cariogenic dental pathogen, was assayed. S. mutans was stored at $-80{ }^{\circ} \mathrm{C}$ as stocks in brain heart infusion broth containing $25 \%(\mathrm{v} / \mathrm{v})$ glycerol. These stocks were streaked onto Mitis Salivarius agar and incubated anaerobically for $48 \mathrm{~h}$ at $37{ }^{\circ} \mathrm{C}$ under an atmosphere of $80 \% \mathrm{~N}_{2}, 10 \% \mathrm{H}_{2}$ and $10 \% \mathrm{CO}_{2}$. Then a single colony of bacteria was inoculated into brain heart infusion broth, and the culture was incubated anaerobically for $12 \mathrm{~h}$ at $37{ }^{\circ} \mathrm{C}$. Bacterial growth was assayed by measurement of absorbance at $600 \mathrm{~nm}$. Optical densities were converted to $\mathrm{CFU} / \mathrm{mL}$ using the conversion $\left(0.64=1 \times 10^{9} \mathrm{CFU} / \mathrm{mL}\right.$ [10]).
Minimum inhibitory concentration (MIC) and minimum bactericidal concentration $(\mathrm{MBC})$ of Far were determined against $S$. mutans using broth microdilution [42]. The initial inoculum was $5 \times 10^{5} \mathrm{CFU} / \mathrm{mL}$, and the concentration of Far ranged from 3.5 to $448 \mu \mathrm{g} /$ $\mathrm{mL}$ with twofold dilutions. Farnesol and chlorhexidine digluconate were assayed in parallel as controls. MIC was defined as the lowest concentration that showed no growth in the medium after anaerobic incubation for $48 \mathrm{~h}$. Brain heart infusion broth on its own was assayed as a blank control, while the same medium supplemented with DMSO $(0.076 \%, \mathrm{v} / \mathrm{v})$ was assayed as a solvent control. MBC was defined as the lowest concentration that showed no surviving bacteria on the agar after incubation for $48 \mathrm{~h}$ at $37^{\circ} \mathrm{C}$. For MBC determination, medium in micropores without bacterial growth was picked up with a sterile inoculating loop and plated onto brain heart infusion blood agar supplemented with 5\% defibrillated sheep blood. These experiments were performed three times.

\section{In vivo anti-caries efficacy of PPi-Far-PMs}

Sprague-Dawley rats were obtained from the Laboratory Animal Center of Southwest Medical University (Luzhou China). All animal experiments were approved by the Animal Ethics Committee of this university (permit 2017060012) and carried out in accordance with the Luzhou municipal government guidelines on animal care and use.

To establish a rat model of dental caries, animals were fed a cariogenic diet with 56\% sucrose (Keyes 2000; Beijing Keao Xieli Feed, Beijing China) and given 5\% sucrose water to drink ad libitum [1, 43]. After weaning, 17-day-old Sprague-Dawley rats (specific pathogen-free) were fed with sodium ampicillin $(0.1 \%$ in food) for 4 days to inhibit endogenous bacterial growth in the oral cavity. Any animals infected with $S$. mutans prior to inoculation were removed from the study on day 21. Then each rat was inoculated with $1 \mathrm{~mL} S$. mutans $\left(7 \times 10^{8} \mathrm{CFU} / \mathrm{mL}\right)$ every day for 7 days. After being checked for infection, 29-day-old rats were randomly divided into nine groups $(n=7)$, and their teeth were topically treated twice daily for 5 weeks with a camel 
hair brush coated with the following treatments: (a) distilled water (negative control), (b) $15 \%$ ethanol (v/v, vehicle control), (c) farnesol (1.10 mg/mL), (d) farnesal (Far; $1.10 \mathrm{mg} / \mathrm{mL})$, (e) chlorhexidine gluconate $(1.10 \mathrm{mg} / \mathrm{mL}$; positive control), (f) blank PMs, (g) Far-PMs (1.10 mg/ $\mathrm{mL}$ Far) and (h) PPi-Far-PMs (1.10 mg/mL Far), ensuring that the drug kept working with the teeth for $1 \mathrm{~min}$. All animals were weighed weekly, and physical appearance was recorded daily. At the end of the 5 weeks, saliva was collected and inoculated onto Mitis Salivarius agar with bacitracin (Sigma) to estimate the $S$. mutans population, and on brain heart infusion agar with $5 \%$ sheep blood to determine the total colony count. Finally, animals were sacrificed and the teeth were collected for further assessment.

To evaluate the anti-caries activity of PPi-Far-PMs, teeth were stained with $0.4 \%$ murexide solution, and caries were scored according to Keyes' system [36]. A stereomicroscope (M205FA, Leica, Germany) was used to assess caries severity on the smooth-surface (Smo), proximal-surface (Pro), and sulcal-surface (Sul). Severity was graded on a four-level scale: enamel affected (E), dentin exposed (Ds), 3/4 of the dentin affected (Dm) and all dentin affected (Dx). Classifications in this study are written in the form "surface-severity", e.g. Smo-E or Sul-Dx. The extent of caries reduction was determined using the following formula:

$$
\begin{aligned}
\text { Caries reduction }(\%)= & (\text { Keyes's score of negative control group } \\
& - \text { Keyes's score of test group }) / \\
& (\text { Keyes's score of negative control group }) \\
& \times 100 \%
\end{aligned}
$$

Rat teeth were collected and assessed in terms of mechanical characteristics and microarchitecture. Compressive strength of teeth was measured using a universal testing machine (Meister E44, USA) at $37 \pm 0.5{ }^{\circ} \mathrm{C}$. A stress-strain curve was recorded as vertical tooth occlusal pressure was applied at $1 \mathrm{~mm} /$ min. After the samples were broken, the compression modulus (slope of stress-strain curve), compressive strength, compressive yield stress, and maximum stress (the highest point of stress-strain curve) were calculated base on the stress-strain curve. Microarchitecture of molars was evaluated using high-resolution micro-computed tomography (micro-CT; skyscan1172, Bruker Corporation). The following parameters were used: voltage, $80 \mathrm{kV}$; current, $80 \mu \mathrm{A}$; exposure time, $2.96 \mathrm{~s}$; scan resolution, $14 \mu \mathrm{m} / \mathrm{slice}$; and total rotation angle, $360^{\circ}$ increasing in $0.5^{\circ}$ increments. Bone mineral density (BMD) and bone volume per tissue volume (BV/TV) were estimated from the three-dimensional reconstructions.

\section{Statistical analysis}

All data were expressed as mean \pm standard deviation (SD) and analyzed using GraphPad Prism 6.0 (GraphPad Software, La Jolla, CA, USA). Differences between treatment groups were analyzed for significance using the Student's $t$ test or Tamhane's T2 test. $P<0.05$ was regarded as statistically significant.

\section{Supplementary information}

Supplementary information accompanies this paper at https://doi. org/10.1186/s12951-020-00633-2.

Additional file 1: Figure S1. ${ }^{1} \mathrm{H}$ NMR and ${ }^{13} \mathrm{C}$ NMR spectra of synthetic compound 1: tert-butyl 2-(2-(2-hydroxyethoxy)acetyl)hydrazine1-carboxylate (HO-PEG-NHNH-Boc). Figure S2. ${ }^{1} \mathrm{H}$ NMR, ${ }^{13} \mathrm{C}$ NMR and ${ }^{31}$ P NMR spectra of synthetic compound 2: sodium 2,2-dimethyl-4,7,13trioxo-3,9,12-trioxa-5,6-diazatetradecan-14-yl hydrogen diphosphate (PPi-PEG-NHNH-Boc). Figure S3. ${ }^{1} \mathrm{H}$ NMR and ${ }^{13} \mathrm{C}$ NMR spectra of synthetic compound 3: sodium 2-(2-(2-hydrazinyl-2-oxoethoxy)ethoxy)-2-oxoethyl hydrogen diphosphate (PPi-PEG-NHNH $\left.{ }_{2}\right)$. Figure S4. ${ }^{1} \mathrm{H}$ NMR and ${ }^{13} \mathrm{C} \mathrm{NMR}$ spectra of synthetic compound 4: sodium 2-oxo-2-(2-(2-oxo-2-(2-((2E,6E)3,7,11-trimethyldodeca-2,6,10-trien-1-ylidene)hydrazinyl)ethoxy)ethoxy) ethyl hydrogen diphosphate (PPi-PEG-hyd-Far)) is available in the online version of this article.

\section{Abbreviations}

$\mathrm{BHI}$ : Brain heart infusion; BMD: Bone mineral density; Boc: t-Butyloxy carbonyl; BV/TV: Bone volume per tissue volume; CHX: Chlorhexidine digluconate; CMC: Critical Micelle Concentration; DCC: N,N'-dicyclohexylcarbodiimide; DMAP. 4-dimethylaminopyridine; Far: Farnesal; Far-PMs: Farnesal-loaded pH-sensitive polymeric micelles; Far-PMs-FITC: FITC-labeled Far-PMs; Hyd: Hydrazone bond; MBC: Minimum bactericidal concentration; MIC: Minimum inhibitory concentration; PMs: Polymeric micelles; PPi: Pyrophosphate; PPi-Far-PMs: PPimediated and farnesal-loaded $\mathrm{pH}$-sensitive dentotropic polymeric micelles; PPi-Far-PMs-FITC: FITC-labeled PPi-Far-PMs; Pro: Proximal-surface; Pro-E: Proximal-surface with enamel affected; SEM: Scanning electron microscopy; SIS: Small intestinal submucosa; Smo: Smooth-surface; Smo-E: Smooth-surface with enamel affected; Sul: Sulcal-surface; Sul-E: Sulcal-surface with enamel affected; Sul-Dm: Sulcal-surface with 3/4 of the dentin affected; Sul-Ds: Sulcalsurface with dentin exposed; Sul-Dx: Sulcal-surface with all dentin affected; TBAP: Tris (tetra-n-butylammonium) hydrogen pyrophosphate.

\section{Acknowledgements}

All authors are thankful for getting help and supports from the following research platforms of the Key Laboratory of Medical Electrophysiology of Ministry of Education, Collaborative Innovation Center for Prevention and Treatment of Cardiovascular Disease, the Drug Discovery Research Center, the Department of Medicinal Chemistry, School of Pharmacy, Southwest Medical University, Luzhou, Sichuan 646000, China.

\section{Authors' contributions}

The study was designed by WL and ZZR. YYP performed the majority of experimental work. CL, LY and LZL performed the HPLC spectroscopy experiments and assisted with the statistical analysis. CZY, LT, WJM and ZZR supervised the project and wrote the manuscript. All authors read and approved the final manuscript.

\section{Funding}

This work was supported by the Sichuan Science and Technology Program (2020YFS0313, 2018JY0237), the Collaborative Fund of Luzhou Government and Southwest Medical University (2019LZXNYDJ21), Key Fund and the Youth Fund and the Transformation Project of Science and Technology Achievements of Southwest Medical University (2018-ZRZD-018, 2017ZRQN-073, 2019ZQN075, 2018002), the Science and Technology Project of the Health Planning Committee of Sichuan (18PJ547), Key Science and Technology Project of Luzhou Government (2018-SYF-19, 2018-RCM-66), the 
National Natural Science Foundation of China (21804112), and the Educational Commission of Sichuan Province (18TD0051).

\section{Availability of data and materials}

All data generated or analyzed during this study are included in this published article and its additional information files.

\section{Ethics approval and consent to participate}

All animal experiments were approved by the Animal Ethics Committee of this university (permit 2017060012) and carried out in accordance with the Luzhou municipal government guidelines on animal care and use.

\section{Consent for publication}

Not applicable.

\section{Competing interests}

The authors declare that they have no competing interests.

Received: 19 February 2020 Accepted: 12 May 2020

Published online: 11 June 2020

\section{References}

1. Pitts NB, Zero DT, Marsh PD, Ekstrand K, Weintraub JA, Ramos-Gomez F, et al. Dental caries. Nat Rev Dis Primers. 2007;12(Suppl. 1):3-6.

2. Jiang $H, H u Y J$, Yang $M, L i u ~ H$, Jiang GS. Enhanced immune response to a dual-promoter anti-caries DNA vaccine orally delivered by attenuated salmonella typhimurium. Immunobiology. 2017;222:730-7.

3. Horev B, Klein MI, Hwang G, Li Y, Kim D, Koo H, et al. pH-activated nanoparticles for controlled topical delivery of farnesol to disrupt oral biofilm virulence. ACS Nano. 2015;9:2390-404.

4. Bowen $\mathrm{WH}, \mathrm{Koo} \mathrm{H}$. Biology of streptococcus mutans-derived glucosyltransferases: role in extracellular matrix formation of cariogenic biofilms. Caries Res. 2011:45:69-86.

5. Flemming $\mathrm{HC}$, Wingender J. The biofilm matrix. Nat Rev Microbiol. 2010;8(9):623-33.

6. Shen S, Samaranayake LP, Yip HK. In vitro growth, acidogenicity and cariogenicity of predominant human root caries flora. J Dent. 2004;32(8):667-78.

7. Agnello M, Cen L, Tran NC, Shi W, McLean JS, He X. Arginine improves pH homeostasis via metabolism and microbiome modulation. J Dent Res. 2017:96:924-30.

8. Kidd EAM, Fejerskov O. What constitutes dental caries? Histopathology of carious enamel and dentin related to the action of cariogenic biofilms. J Dent Res. 2004;83(Spec Issue C):35-8.

9. Bowen WH. Dental caries - not just holes in teeth! A perspective. Mol. Oral Microbiol. 2016;31:228-33.

10. Chen F, Wang D. Novel technologies for the prevention and treatment of dental caries: a patent survey. Expert Opin Ther Pat. 2010;20(5):681-94.

11. Selwitz RH, Ismail Al, Pitts NB. Dental caries. Lancet. 2007;369(Issue 9555):51-9.

12. Jayasree R, Kumar TSS, Mahalaxmi S, Abburi S, Rubaiya Y, Doble M. Dentin remineralizing ability and enhanced antibacterial activity of strontium and hydroxyl ion co-releasing radiopaque hydroxyapatite cement. J Mater Sci Mater Med. 2017;28:95.

13. ten Cate JMB. The need for antibacterial approaches to improve caries control. Adv Dent Res. 2009;21(1):8-12.

14. Kawarai T, Narisawa N, Yoneda S, Tsutsumi Y, Ishikawa J, Hoshino Y, et al. Inhibition of streptococcus mutans biofilm formation using extracts from Assam tea compared to green tea. Arch Oral Biol. 2016;68:73-82.

15. Liu C, Worthington RJ, Melander C, Wu H. A new small molecule specifically inhibits the cariogenic bacterium streptococcus mutans in multispecies biofilms. Antimicrob Agents Chemother. 2011;55(6):2679-87.

16. Zhang TT, Guo HJ, Liu XJ, Chu JP, Zhou XD. Galla chinensis compounds remineralize enamel caries lesions in a rat model. Caries Res. 2016;50:159-65.

17. Koo H, Schobel B, Scott-Anne K, Watson G, Bowen WH, Cury JA, et al. Apigenin and tt-farnesol with fluoride effects on S. mutans biofilms and dental caries. J Dent Res. 2005;84(11):1016-20.

18. Jeon JG, Pandit S, Xiao J, Greqoire S, Falsetta ML, Klein Ml, et al. Influences of trans-trans farnesol, a membrane-targeting sesquiterpenoid, on Streptococcus mutans physiology and survival within mixed-species oral biofilms. Int. J. Oral Sci. 2011;3(2):98-106.
19. Jabra-Rizk MA, Meiller TF, James CE, Shirtliff ME. Effect of farnesol on staphylococcus aureus biofilm formation and antimicrobial susceptibility. Antimicrob Agents Chemother. 2006;50(4):1463-9.

20. Pammi M, Liang R, Hicks JM, Barrish J, Versalovic J. Farnesol decreases biofilms of Staphylococcus epidermidis and exhibits synergy with nafcillin and vancomycin. Pediatr Res. 2011;70:578-83.

21. Kong EF, Tsui C, Kucharíková S, Van Dijck P, Jabra-Rizk MA. Modulation of Staphylococcus aureus response to antimicrobials by the candida albicans quorum sensing molecule farnesol. Antimicrob Agents Chemother. 2017;61:e01573.

22. Chen F, Jia Z, Rice KC, Reinhardt RA, Bayles KW, Wang D. The development of drug-free therapy for prevention of dental caries. Pharm Res. 2014;31(11):3031-7.

23. Chen F, Jia Z, Rice KC, Reinhardt RA, Bayles KW, Wang D. The development of dentotropic micelles with biodegradable tooth-binding moieties. Pharm Res. 2013;30(11):2808-17.

24. Krishnan $\mathrm{V}$, Bhatia A, Varma H. Development, characterization and comparison of two strontium doped nano hydroxyapatite molecules for enamel repair/regeneration. Dent Mater. 2016;32:646-59.

25. Wang XY, Cai Q, Zhang XH, Wei Y, Xu MM, Yang XP, et al. Improved performance of Bis-GMA/TEGDMA dental composites by net-like structures formed from SiO2 nanofiber fillers. Mater Sci Eng C Mater Biol Appl. 2016;59:464-70.

26. Koo H, Rosalen PL, Cury JA, Bonwen WH. Effects of compounds found in propolis on Streptococcus mutans growth and on glucosyltransferase activity. Antimicrob Agents Chemother. 2002;46(5):1302-9.

27. Ulbrich K, Holá K, Šubr V, Bakandritsos A, Tuček J, Zbořil R. Targeted drug delivery with 28-polymers and magnetic nanoparticles: covalent and noncovalent approaches, release control, and clinical studies. Chem Rev. 2016;116:5338-431.

28. DeBenedictis EP, Hamed E, Keten S. Mechanical reinforcement of proteins with polymer conjugation. ACS Nano. 2016;10:2259-67.

29. Zhang X, Wang H, Ma Z, Wu B. Effects of pharmaceutical PEGylation on drug metabolism and its clinical concerns. Expert Opin Drug Metab Toxicol. 2014;10(12):1691-702.

30. Li HM, Sun X, Zhao D, Zhang ZR. A cell-specific poly(ethylene glycol) derivative with a wheat-like structure for efficient gene delivery. Mol Pharm. 2012;9:2974-85

31. Li XJ, Takashima M, Yuba E, Harada A, Kono K. PEGylated PAMAM dendrimerdoxorubicin conjugate-hybridized gold nanorod for combined photothermal-chemotherapy. Biomaterials. 2014;35:6576-84.

32. Yilmaz G, Demir B, Timur S, Becer CR. Poly(methacrylic acid)-coated gold nanoparticles: functional platforms for theranostic applications. Biomacromology. 2016;17:2901-11.

33. Etrych T, Sírová M, Starovoytova L, Ríhová B, Ulbrich K. HPMA copolymer conjugates of paclitaxel and docetaxel with $\mathrm{pH}$-controlled drug release. Mol. Pharmaceutics. 2010;7(4):1015-26.

34. Wang $Q$, Sun $X$. Recent advances in nanomedicines for the treatment of rheumatoid arthritis. Biomater Sci. 2017;5:1407-20.

35. Falsetta ML, Klein MI, Lemos JA, Salva BB, Aqidi S, Scott-Anne KK, et al. Novel antibiofilm chemotherapy targets exopolysaccharide synthesis and stress tolerance in Streptococcus mutans to modulate virulence expression in vivo. Antimicrob Agents Chemother. 2012;56(12):6201-11.

36. Keyes PH. Dental caries in the molar teeth of rats: II A method for diagnosing and scoring several types of lesions simultaneously. J Dent Res. 1958;37(6):1088-99.

37. Wang G, Wang JJ, Chen XL, Du L, Li F. Quercetin-loaded freeze-dried nanomicelles: improving absorption and anti-glioma efficiency in vitro and in vivo. J Control Rel. 2016;235:276-90.

38. Wang YL, Luo YL, Li CH, Zhang XQ, Pi C, Yu L, et al. Optimized formulation of multivesicular liposomes loaded with oleanolic acid enhanced anticancer effect in vitro. Drug Des Dev Ther. 2017;11:955-68.

39. Wang JX, Xu WG, Li SX, Qiu HP, Li ZB, Wang CX, Wang XQ, Ding JX. Polylactide-cholesterol stereocomplex micelle encapsulating chemotherapeutic agent for improved antitumor efficacy and safety. J Biomed Nanotechnol. 2018;14:2102-13.

40. Luo JC, Chen W, Chen XH, Qin TW, Huang YC, Xie HQ, et al. A multi-step method for preparation of porcine small intestinal submucosa (SIS). Biomaterials. 2011;32(3):706-13.

41. Bian TR, Zhao K, Meng QN, Tang YF, Jiao H, Luo J, et al. Synthesis of plate-like single-crystal hydroxyapatite rods with c-axis orientation by biotemplate small intestinal submucosa. Ceram Int. 2017:43(15):11807-14. 
42. Xu X, Zhou XD, Wu CD. The tea catechin epigallocatechin gallate suppresses cariogenic virulence factors of streptococcus mutans. Antimicrob Agents Chemother. 2011;5(3):1229-36.

43. Yucesoy DT, Fong H, Gresswell C, Saadat S, Chung WO, Dogan S, et al. Early caries in an in vivo model: structural and nanomechanical characterization. $J$ Dent Res. 2018;97:1452-9.

\section{Publisher's Note}

Springer Nature remains neutral with regard to jurisdictional claims in published maps and institutional affiliations.
Ready to submit your research? Choose BMC and benefit from:

- fast, convenient online submission

- thorough peer review by experienced researchers in your field

- rapid publication on acceptance

- support for research data, including large and complex data types

- gold Open Access which fosters wider collaboration and increased citations

- maximum visibility for your research: over $100 \mathrm{M}$ website views per year

At BMC, research is always in progress.

Learn more biomedcentral.com/submissions 